\title{
Genome-wide identification and gene expression analysis of Clade $A$ protein phosphatase 2C family genes in Brassica juncea var. tumida
}

Chunhong Cheng, Zhaoming Cai, Rongbin Su, Yuanmei Zhong, Li Chen, Lixia Wang, Guojian Chen, Lun Yan and Changman $\mathrm{Li}^{*}$

Chongqing key Laboratory for Conservation and Utilization of Characteristic Plant Resources in Wuling Mountain Area, School of Advanced Agriculture and Bioengineering, Yangtze Normal University, Chongqing 408100, P.R. China.

Corresponding Author:

Changman Li

Juxian Road, Fuling, Chongqing, 408100, P.R. China

Email address: cmlyznu@163.com

\begin{abstract}
Abscisic acid (ABA) plays crucial roles in plant response to environmental stresses and development. The clade A phosphatases (PP2Cs) play a crucial role in ABA signaling. However, little is known about the details regarding PP2Cs family genes in Brassica juncea var. tumida. Here, 20 clade A PP2Cs family genes were identified in tuber mustard genome, including BjuABIls, BjuABI2s, BjuAHG1s, BjuAHG3s, BjuHAB1, BjuHAB2s, BjuHAI1s, BjuHAI2s and BjuHAI3. The promoters of BjuPP2Cs family genes contained various of cis-acting elements, such as ABRE, GT1GMSCAM4, ARFAT and MYB1AT. We also analyzed the expression pattern of clade A BjuPP2Cs under abiotic stresses (low temperature, $\mathrm{NaCl}$ and $\mathrm{ABA}$ ) treatment, pathogen Plasmodiophora brassicae treatment and different stages of stem swollen. The results suggested that clade A BjuPP2Cs regulated tuber mustard response to $P$. brassicae to mediate the formation of clubroot and might play roles in stem swelling and response to abiotic stresses . This study provides valuable information for further functional investigations of clade A PP2Cs family genes in B. juncea var. tumida.
\end{abstract}


Keywords Brassica juncea var. tumida, clade A BjuPP2Cs, abiotic stresses, Plasmodiophora brassicae, stem swollen

Abbreviations

ABA

Abscisic acid

PP2C

Protein Phosphatase 2C

ABI1

ABA INSENSITIVE 1

$\mathrm{ABI} 2$

ABA INSENSITIVE 2

AHG1

ABA-HYPERSENSITIVE GERMINATION 1

AHG3

ABA-HYPERSENSITIVE GERMINATION 3

HAB 1

HYPERSENSITIVE TO ABA1

HAB2

HOMOLOGY TO ABI2

HAI1

HIGHLY ABA-INDUCED PP2C GENE 1

HAI2

HIGHLY ABA-INDUCED PP2C GENE 2

HAI3

HIGHLY ABA-INDUCED PP2C GENE 3

PPs Protein Phosphatases

PPP Phosphor-Protein Phosphatase

PPM Phosphoprotein Metallophosphatases

SnRK2s Sucrose Nonfermenting-1-related Protein Kinase Class 2 


\section{Introduction}

The reversible phosphorylation of proteins by protein phosphatases is a crucial process which modulates plant growth and development ${ }^{[1,2]}$. Protein phosphatases (PPs), removed the phosphate group of phosphorylated proteins, play important roles in regulation of protein function. PPs can be divided into two major subfamilies: protein tyrosine phosphatases and protein serine/threonine phosphatases. Based on the distinct amino acid sequences and crystal structures, the protein serine/threonine phosphatases can be classified into two groups: the phosphor-protein phosphatase (PPP) and the phosphoprotein metallophosphatases (PPM). The PP1, PP2A and PP2B subgroups belong to PPP family, whereas the PP2C subgroup belongs to the PPM family $\left(\mathrm{Mg}^{2+} \text { or } \mathrm{Mn}^{2+} \text { dependent }\right)^{[3]}$.

ABA plays a crucial role in plant response to environmental stresses such as abiotic stresses (salinity and low temperature stresses) and biotic stresses (such as pathogen) [4-6]. Until now, the core components of the ABA signaling pathway include pyrabactin resistance (PYR)/PYR1-like (PYL) regulatory components of ABA receptor (RCAR) protein family (ABA receptors), the co-receptors clade A protein type 2C phosphatases (PP2Cs), and sucrose nonfermenting-1-related protein kinase class 2 (SnRK2s). In the absence of ABA, PP2Cs interact with SnRK2s to removing the phosphate group and inhibit the kinase activity of SnRK2s, which resulted in turning off $\mathrm{ABA}$ signaling pathway. In the presence of $\mathrm{ABA}, \mathrm{ABA}$ binds the $\mathrm{PYR} / \mathrm{PYL} / \mathrm{RCAR}$ receptors and then interacts with PP2Cs, which inhibit the phosphatase activity of PP2Cs and eliminate the inhibitory effect of the phosphatases on SnRK2s to turn on the ABA signaling [7-9]. In Arabidopsis, nine clade A PP2Cs

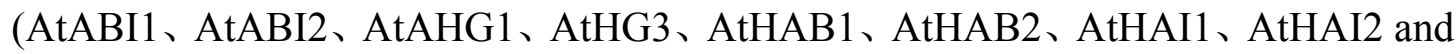
AtHAI3) have been identified as the negative regulators in ABA signaling pathway. The dominant mutations abil-1 and abi2-1 showed the insensitive phenotype to ABA in germination and greening stages indicating that $\mathrm{ABI} 1$ and $\mathrm{ABI} 2$ were the negative regulators in ABA signaling [10]. The ahgl-1 and ahg3-1 mutant showed ABA hypersensitivity phenotype in germination and post germination growth stages, but 
not in adult plants. And the expression level of $A H G 1$ was strongest in seeds indicating that it played an important role in response to ABA in seed [11,12]. HAB1 underwent alternative splicing and produced two splice variants, named HAB1.1 and $H A B$ 1.2. HAB1.1 and HAB1.2 played the opposing roles in ABA-mediated seed germination and post-germination stages [13]. In B.rapa the PP2C genes were structurally conserved based on the amino acid sequence alignment, phylogenetic analysis and conserved domains; and the gene expression levels were induced by heat, cold, ABA and drought treatment ${ }^{[14]}$. Twenty group A PP2C homologous genes of $B$. oleracea were identified; the genetic analysis corroborated the presence of two to three gene copies in B. oleracea in comparison to clade A PP2C genes in Arabidopsis thaliana; the gene expression patterns of $P P 2 C s$ in $B$. oleracea were significant differences ${ }^{[15]}$.

Tuber mustard, Brassica juncea var. tumida (AABB, $2 \mathrm{n}=36)$, an allotetraploid species, which belongs to Brassicaceae, came from a natural cross between B. rapa $(\mathrm{AA}, 2 \mathrm{n}=20)$ and B. nigra $(\mathrm{BB}, 2 \mathrm{n}=16)$, followed by chromosome doubling ${ }^{[16]}$. The swollen stem of tuber mustard is the raw material of Fuling mustard and is an important vegetable. However, little is known about the regulation mechanism of stem swelling [17]. And during the growth and development stages, tuber mustard frequently suffers from abiotic and biotic stresses, such as salt stress, cold stress and Plasmodiophora brassicae, which leads to the suppression of plant growth and development and the formation of clubroot. Clubroot is one of the most serious biotic stresses that plants need to cope with and usually results in the suppression of tuber mustard growth and stem swollen, leading to limitation in yield ${ }^{[18]}$. Therefore, illustrating the mechanisms underlying the stem swollen and the resistance to abiotic and biotic stresses will be helpful for improving the production of tuber mustard. Clade A PP2Cs of ABA signaling pathway play important roles in response to abiotic and biotic stresses and regulation plant development. However, the gene structures, protein motifs, gene duplication events and functions of clade A PP2Cs in B. juncea var. tumida remains mainly unknown.

In this study, twenty clade A PP2C family genes were identified in $B$. juncea var. 
tumida genome. The phylogenic relationship, gene structures, and protein motifs were compared between clade A BjuPP2Cs and AtPP2Cs and found that they shared similar gene characteristics. Following the analysis of gene features, we analyzed the transcript levels of clade A BjuPP2Cs under P. Brassicae, $\mathrm{NaCl}$, low temperature and ABA treatment and different stages of stem swollen. The results showed that clade A BjuPP2Cs were induced by pathogen and abiotic stresses and induced in the stages of stem swollen, suggesting that clade A BjuPP2C family genes played crucial roles in plant response to abiotic stresses, pathogen P. Brassicae and regulation of stem swollen.

\section{Materials and Methods}

\section{Materials and growth conditions}

The tuber mustard cultivar Yong'an was used in this study. The seeds were sterilized and sowed in MS medium. The growth room was at $22^{\circ} \mathrm{C}$ and 6000 lx (long-day conditions; $16 \mathrm{~h}$ light/ $8 \mathrm{~h}$ dark). To analyze the gene expression patterns of clade A BjuPP2Cs genes under biotic stress, 14-day-old seedlings were treated with $P$. brassicae liquid $\left(\mathrm{OD}_{600}=0.07\right)$ for $0,0.25,0.5,1,3,5,7$, and 9 days. To analysis the gene expression level in different growth stages of stem swelling, the samples D1 (the stems of 1-month-old seedlings, six leaf stage), D2 (the stems of 2-month-old seedlings, primary stage of stem swelling), D3 (the stems of 3-month-old seedlings, early stage of stem swelling), D4 (the stems of 4.5-month-old seedlings, fast-growing stage of stem swelling) and D5 (the stems of 5-month-old seedlings, last stage of stem swelling) of B. juncea var. tumida which were grown in the field were collected. To analyze the gene expression patterns of clade A BjuPP2Cs genes under abiotic stresses, 5-day-old seedlings were treated with $50 \mu \mathrm{M} \mathrm{ABA}, 100 \mathrm{mM} \mathrm{NaCl}$ and $4{ }^{\circ} \mathrm{C}$ treatment for $3 \mathrm{~h}$.

\section{Bioinformatics analysis}

The gene sequences of clade A AtPP2Cs and their homologous genes in tuber mustard were searched in TAIR (http://www.arabidopsis.org/) and Brassica 
Databases (http://brassicadb.cn/\#/). The phylogenic tree was analysis with the neighbor-joining method by MEGA5. Gene structures were analyzed by Gene Structure Display Server 2.0 (http://gsds.cbi.pku.edu.cn/). The promoter regions of clade A BjuPP2Cs were chose by Flanking region in Brassica Database, and the $2 \mathrm{~kb}$ regions upstream of ATG contained no other genes. So the regions located $2 \mathrm{~kb}$ upstream of the clade A BjuPP2Cs coding sequences were used as the promoter sequences, and promoter cis-element analysis was performed using New PLACE (https://www.dna.affrc.go.jp/PLACE/?action=newplace). Protein domain was analysis by SMART (http://smart.embl-heidelberg.de/),

ExPASy (http://prosite.expasy.org/prosite.html) and ESPrint 3.0 (https://espript.ibcp.fr/ESPript/cgi-bin/ESPript.cgi).

\section{Gene expression analysis}

Total RNA of tuber mustard was extracted under pathogen and abiotic stresses treatment. qRT-PCR was performed using the cDNA. The transcript level was analysis by the comparative CT method, and BjuActin3 was used as the internal reference. The qRT-PCR experiments were carried out three times with three replicates each. The primers used in this study were listed in Table S1.

\section{Statistical analysis}

All data were analyzed using SigmaPlot 10.0 (Systat Software, Inc., Chicago, IL) and SPSS 16.0 software. The averages and standard deviations of all results were calculated, and for multiple groups of samples, the one-way ANOVA followed by the Dunnett test was used. The statically significant treatments were marked with '***, $(\mathrm{P}<0.001),{ }^{* *}$ ' $(0.001<\mathrm{P}<0.01)$ and '*’ $(0.01<\mathrm{P}<0.05)$.

\section{Results}

Genome-wide identification and characterization of clade A BjuPP2Cs in B. juncea var. tumida

Twenty genes as homologs of clade A PP2Cs were identified in B. juncea var. tumida through BLASTP in Brassica database using nine clade A AtPP2C protein sequences 
as references, and the naming rules based on the names of PP2Cs in Arabidopsis thaliana, such as the five copies of AtABI1 named BjuABI1-1, BjuABI1-2, BjuABI1-3, BjuABI1-4 and BjuABI1-5 (Table 1). The gene lengths ranged from 1288 bp to 5344 bp with 2-8 exons in each sequence. The protein lengths of these twenty PP2C homologs ranged from 325 (BjuABI1-4) to 606 (BjuAHG3-3) amino acid (aa) residues. The relative molecular weights of those proteins varied from $35.907 \mathrm{kD}$ (BjuABI1-4) to $67.615 \mathrm{kD}$ (BjuAHG3-3), and the isoelectric point (PI) ranged from 4.40 to 7.51 (Table 1). The twenty $B j u P P 2 C$ genes were distributed in 13 of the 18 chromosomes of B. juncea var. tumida. Each of the chromosomes A01, A05, $\mathrm{A} 08, \mathrm{~A} 09, \mathrm{~B} 01, \mathrm{~B} 05, \mathrm{~B} 06, \mathrm{~B} 07$, and $\mathrm{B} 08$ contained one gene, each of the chromosomes A03 and B02 contained two genes, and each of the chromosomes A10 and B03 contained three genes (Figure 1). 
Table 1. The clade A BjuPP2Cs family members in $B$. juncea var. tumida.

\begin{tabular}{|c|c|c|c|c|c|c|c|c|c|c|c|}
\hline Group & Gene name & Lous & Sequence ID & Exon & Start (bp) & End (bp) & Genomics (bp) & CDS (bp) & Protein (aa) & pI & MW (kD) \\
\hline \multirow[t]{5}{*}{$A t A B I 1$} & BjuABI1-1 & A01 & ВјиА004393 & 4 & 9995166 & 9996741 & 1576 & 1248 & 415 & 6.44 & 45.7 \\
\hline & BjuABI1-2 & A03 & BjuA013512 & 4 & 33638598 & 33640105 & 1508 & 1233 & 411 & 5.51 & 45.158 \\
\hline & BjuABI1-3 & B05 & ВjuB040327 & 4 & 7461353 & 7462895 & 1543 & 1257 & 419 & 5.87 & 45.785 \\
\hline & BjuABI1-4 & A08 & ВjuA029591 & 3 & 18441423 & 18442925 & 1503 & 975 & 325 & 6.15 & 35.907 \\
\hline & BjuABI1-5 & B03 & ВјиB043703 & 3 & 5139792 & 5141317 & 1526 & 1209 & 402 & 5.92 & 44.679 \\
\hline \multirow[t]{2}{*}{$A t A B I 2$} & BjuABI2-1 & B02 & ВjuB001428 & 8 & 22604047 & 22608170 & 4124 & 1614 & 538 & 5.14 & 63.057 \\
\hline & BjuABI2-2 & A 10 & ВјuA044909 & 8 & 10610992 & 10616335 & 5344 & 1611 & 537 & 7.48 & 59.467 \\
\hline \multirow[t]{2}{*}{$A t A H G 1$} & ВjuAHG1-1 & A03 & ВjuA010184 & 4 & 8253750 & 8255933 & 2184 & 1257 & 418 & 6.08 & 45.533 \\
\hline & ВjuAHG1-2 & B08 & ВjuB041185 & 4 & 15899473 & 15901555 & 2083 & 1275 & 424 & 6.08 & 46.768 \\
\hline \multirow[t]{3}{*}{ AtAHG3 } & BjuAHG3-1 & A05 & ВjuA020514 & 2 & 28490862 & 28492149 & 1288 & 1200 & 400 & 6.04 & 43.061 \\
\hline & BjuAHG3-2 & B07 & ВjuB006899 & 3 & 3123674 & 3125019 & 1346 & 1170 & 389 & 6.22 & 42.243 \\
\hline & BjuAHG3-3 & B01 & ВjuB023632 & 3 & 42663804 & 42668223 & 4420 & 1818 & 606 & 7.51 & 67.615 \\
\hline AtHAB1 & BjuHAB1 & B03 & ВјuB030059 & 3 & 39259177 & 39260975 & 1799 & 1137 & 379 & 4.63 & 40.875 \\
\hline AtHAB2 & ВјuHAB2 & B03 & ВjuB004338 & 2 & 2791346 & 2793227 & 1882 & 1158 & 386 & 4.40 & 42.644 \\
\hline \multirow[t]{2}{*}{ AtHAI1 } & ВjuHAI1-1 & A 10 & BjuA038574 & 4 & 11316559 & 11318415 & 1857 & 1236 & 412 & 6.07 & 45.517 \\
\hline & ВjuHAI1-2 & B02 & ВjuB008108 & 4 & 29546261 & 29548082 & 1822 & 1194 & 397 & 7.06 & 44.176 \\
\hline \multirow[t]{3}{*}{ AtHAI2 } & ВjuHAI2-1 & A09 & BjuA019471 & 3 & 54391632 & 54393366 & 1735 & 1308 & 436 & 6.17 & 48.931 \\
\hline & ВjuHAI2-2 & B06 & ВjuB015401 & 3 & 17704399 & 17706077 & 1679 & 1263 & 421 & 6.17 & 47.211 \\
\hline & ВjuHAI2-3 & A10 & ВjuA037326 & 3 & 782631 & 784079 & 1449 & 1281 & 426 & 5.93 & 47.804 \\
\hline AtHAI3 & ВjuHAI3 & Contig & ВјиO013253 & 3 & 1153566 & 1155192 & 1627 & 1104 & 368 & 5.50 & 41.009 \\
\hline
\end{tabular}

pI: Isoelectric point; $\mathrm{MW}$ : molecular weight; CDS: coding sequence. 


\section{Phylogenic analysis and gene structures of clade A PP2C family genes}

To analyze the evolutionary relationships between PP2C homologs in B. juncea var. tumida and Arabidopsis thaliana, a phylogenetic tree was constructed by MEGA5 software using the neighbor-joining method. According to the phylogenic tree, twenty clade A BjuPP2Cs with nine AtPP2Cs were identified and clustered into nine clades. The first clade was AtHAI2 and three homologs BjuHAI2-1, BjuHAI2-2 and BjuHAI2-3; the second clade was AtHAI3 and BjuHAI3; the third clade was AtHAI1 and its two homologs BjuHAI1-1 and BjuHAI1-2; AtAHG3 and its homologs BjuAHG3-1, BjuAHG3-2 and BjuAHG3-3 were clustered into one clade; AtAHG1, BjuAHG1-1 and BjuAHG1-2 were clustered into one clade; AtHAB1 and BjuHAB1 were clustered into one clade; AtHAB2 and BjuHAB2 were clustered into one clade; the eighth clade was AtABI2 and its two homologs BjuABI2-1 and BjuABI2-2; and the last clade was AtABI1 and its homologs BjuABI1-1, BjuABI1-2, BjuABI1-3, BjuABI1-4 and BjuABI1-5 (Figure 2). Clade A PP2C family genes in the same subfamilies may have similar functions. To understand their gene structures, we analyzed the gene exon-introns. According to the results, AtHAII and BjuHAIls, AtHAI2 and BjuHAI2s, AtHAI3 and BjuHAI3s, AtAHG1 and BjuAHG1s had the same gene structures, respectively; AtAHG3 contained 4 exons, while BjuAHG3-1, BjuAHG3-2 and BjuAHG3-3 had 2, 3 and 3 exons, respectively; AtHAB1 and $B j u H A B 1$ contained 4 and 3 exons, respectively; AtHAB2 and BjuHAB2 contained 4 and 2 exons, respectively; BjuABI2-1 and BjuABI2-2 all contained 8 exons with the exception of AtABI2 (4 exons); AtABI1, BjuABI1-1, BjuABI1-2 and BjuABI1-3 all had 4 exons, in contrast, BjuABI1-4 and BjuABI1-5 contained 3 exons (Figure 2).

\section{The conserved motif analysis of clade A PP2C homologs}


To analysis the protein function of $\mathrm{PP} 2 \mathrm{C}$ homologs, protein conserved motifs were conducted. The results showed that all the clade A BjuPP2Cs contained PP2Cc domain (Serine/threonine phosphatases, family2C, catalytic domain), which was consistent with clade A AtPP2Cs; only BjuABI2-1 and BjuABI2-2 contained transmembrane domain (Figure 3). As some clade A BjuPP2Cs lacked of some exons, such as BjuABIIs and BjuAHG3s, might lead to the different effects on the catalytic PP2C domain in their proteins. So we analyzed the motif sequences of BjuPP2Cs by ClustalX and ESPrint 3.0 (https://espript.ibcp.fr/ESPript/cgi-bin/ESPript.cgi). The protein serine/threonine phosphatase 2C (PP2C) family contained 11 conserved motif, named motif $1-5$, motif $5 \mathrm{a}$, motif $5 \mathrm{~b}$ and motif $6-11^{[19]}$. In our result, all of the 11 conserved motifs in the clade A BjuPP2C proteins were identified, suggesting that the catalytic PP2C domain in their proteins were conserved even if they had different gene structures (Fig. S1).

\section{Promoter cis-acting regulatory elements prediction of clade A BjuPP2C homologs}

To further understand the potential roles of clade A BjuPP2Cs, the promoters of clade A BjuPP2Cs were analyzed. The promoter regions of clade A BjuPP2Cs were chose by Flanking region in Brassica Database, and the $2 \mathrm{~kb}$ regions upstream of ATG contained no other genes. So we chose the $2000 \mathrm{bp}$ DNA fragment upstream of the ATG start codon as the promoter sequences and analyzed promoter cis-elements. The results showed that all BjuPP2Cs contained at least one hormone-related elements in the promoters such as ARFAT (TGTCTC, responsive to auxin) [20] and

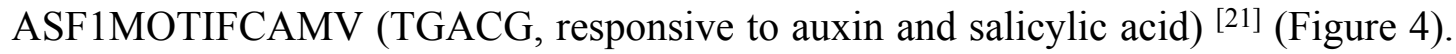
The promoters of all BjuPP2Cs genes, except BjuHAI1-2, contained 1 to 7 cis-acting elements related to ABA, such as ABRE (ACGTG, responsive to Abscisic acid, ABA) [22] (Figure 4). In addition, the promoters of the BjuPP2Cs homologs all contained stressed-related elements, such as the MYCCONSENSUSAT (CANNTG, responsive 
to dehydration stress) ${ }^{[23]}$, MYBATRD22 (CTAACCA, responsive to dehydration stress) [24], MYB1AT (WAACCA, responsive to dehydration stress) [23], CBFHV (RYCGAC, responsive to dehydration stress) ${ }^{[25]}$, CRTDREHVCBF2 (GTCGAC, responsive to low temperature) ${ }^{[26]}$, LTRECOREATCOR15 (CCGAC, responsive to low temperature) ${ }^{[27]}$, GT1GMSCAM4 motif (GAAAAA, responsive to pathogen and salt stress) ${ }^{[28]}$, GCCCORE (GCCGCC, responsive to pathogen) ${ }^{[29]}$ and MYB1LEPR (GTTAGTT, responsive to defence) ${ }^{[30]}$ (Figure 4). Together, the promoters of BjuPP2Cs homologs contained diverse cis-elements responsive to $\mathrm{ABA}$, auxin, dehydration, low temperature and pathogen, indicating that the genes expression of clade A BjuPP2Cs homologous were regulated by hormone, biotic stresses and abiotic stresses, and clade A BjuPP2Cs might play a role in regulating tuber mustard response to hormone and stresses.

\section{Tissue specific expression pattern analysis of clade A BjuPP2Cs}

To investigate the tissue specific expression patterns of clade A BjuPP2Cs, we analyzed the gene expression levels at different growth stages and tissues (root, stem, swollen stem, leaf, flower and pod) using qRT-PCR. According to the results, clade A $B j u P P 2 C s$ were expressed in multiple tissues. Interestingly, the expression levels of BjuABI1-1, BjuABI1-5, ВjuABI2-1, ВjuABI2-2, BjuAHG1-1, BjuAHG3-3, BjuHAI1-1, BjuHAI2-1, BjuHAI2-3 and BjuHAI3 showed significant difference between stem and swollen stem, indicating that these clade A BjuPP2Cs might play a role in the regulation of stem swollen (Figure 5). The expression levels of BjuABI1-2, BjuABII-3, BjuABI1-4, BjuAHG3-3, BjuHAB1, BjuHAB2, BjuHAI1-1 and BjuHAI2-1 were higher in root than that in other organs. Almost all the clade A BjuPP2Cs were expressed very low in flower and pod, especially BjuHAI2-1 and BjuHAI2-2 (nearly no expression) (Figure 5). The expression levels of the clade A BjuPP2Cs varied in different tissues and organs, indicating that they might play different roles in different organs. In addition, the different expression patterns of the same gene in different 
tissues and organs suggested that the expression patterns of the genes were existence of space-time specificity.

\section{The expression patterns of clade A BjuPP2Cs in B. juncea var. tumida under}

\section{abiotic stresses}

To further analysis the expression levels of clade A BjuPP2Cs under abiotic stresses treatment, qRT-PCR assays were performed using 5-day-old tuber mustard seedlings treated with $50 \mu \mathrm{M} \mathrm{ABA}, 100 \mathrm{mM} \mathrm{NaCl}$ and $4{ }^{\circ} \mathrm{C}$ treatment for $3 \mathrm{~h}$. The qRT-PCR results showed that all of the clade A BjuPP2Cs genes were induced by $\mathrm{NaCl}$ significantly (Figure 6). Under low temperature treatment, the expression levels of BjuABI1-2 to BjuABI1-4, BjuABI2-2, BjuAHG1-2, BjuHAB2, BjuHAI2-3 and BjuHAI3 were induced, whereas, the expression levels of BjuABI2-1, BjuAHG3-1 to BjuAHG3-3 and BjuHAI2-1 were suppressed (Figure 6). Under ABA treatment, BjuABI1-1 to BjuABI1-4, BjuABI2-2, BjuAHG1-2, BjuAHG3-2, BjuAHG3-3, BjuHAB1, BjuHAI1-2 and BjuHAI2-3 were induced by ABA treatment, however, BjuABI2-1, BjuAHG3-1 and BjuHAB2 were inhibited (Figure 6).

The expression patterns of clade A BjuPP2Cs in $B$. juncea var. tumida under $P$. brassicae stress

To further analysis the gene expression patterns of clade A BjuPP2Cs in tuber mustard under pathogen stress, qRT-PCR assays were performed using tuber mustard seedlings treated with $P$. brassicae. Under $P$. brassicae treatment, 2-week-old tuber mustard seedlings were treated with $P$. brassicae for $0,0.25,0.5,1,3,5,7$ and 9 days, and the qRT-PCR results showed that BjuABI1-5, BjuAHG1-1, BjuAHG3-2, BjuAHG3-3, BjuHAB1, ВјuHAB2, BjuHAI1-1, BjuHAI2-1, BjuHAI2-3 and BjuHAI3 were highly induced by pathogen, especially on day 0.5 after pathogen treatment. The 
result indicated that BjuABI1-5, BjuAHG1-1, BjuAHG3-2, BjuAHG3-3, BjuHAB1, BjuHAB2, BjuHAI1-1, BjuHAI2-1, BjuHAI2-3 and BjuHAI3 may play roles in P. brassicae tolerance (Figure 7).

\section{Gene expression levels of clade A BjuPP2Cs in B. juncea var. tumida during stem} swelling stages

The swollen stem is the raw material of "Fuling Mustard", however, the regulation mechanism of stem swelling was still undiscovered. To further explore the roles of clade A BjuPP2Cs in regulating the formation and development of swollen stem in tuber mustard, we collected the stems of B. juncea var. tumida, which were taken in the field at different growth and development stages. The stems were named as D1 (the stems of 1 month old seedlings, six leaf stage), D2 (the stems of 2 month old seedlings, primary stage of stem swelling), D3 (the stems of 3 month old seedlings, early stage of stem swelling), D4 (the stems of 4.5 month old seedlings, fast growing stage of stem swelling), and D5 (the stems of 5 month old seedlings, last stage of stem swelling). The qRT-PCR assay was performed and the results showed that the gene expression levels of BjuABI1-1, BjuAHG1-1, BjuHAI2-2, BjuHAI2-3 and BjuHAI3 were inhibited during stem swelling stages (D2-D4 stages). The expression levels of BjuABI1-3, BjuABI2-1, BjuHAB1 and BjuHAI1-1 were induced during stem swelling stages (D2-D4 stages) (Figure 8). The results suggested that clade A BjuPP2Cs may play roles in the regulation of the formation and development of swollen stem.

\section{Discussion}

The swollen stem of B. juncea var. tumida is the raw material of Fuling mustard, which is an important economic vegetable in China and famous for its special flavour and nutritional value ${ }^{[17]}$. P. brassicae leads to the formation of clubroot in tuber mustard, which is one of the most serious stresses and results in the inhibition in plant growth and stem swollen, and leading to limitation in yield ${ }^{[18,31]}$. Clade A PP2C family genes play important roles in the regulation of plant development and tolerance 
to stress, however, the identity and expression patterns of B. juncea var. tumida clade A PP2C genes are unknown. This study is the first identification of clade A BjuPP2Cs in B. juncea var. tumida and adds a new layer to the function of clade A BjuPP2Cs.

In this study, twenty clade A BjuPP2Cs genes were identified and located in 13 of 18 chromosomes based on the nine clade A AtPP2Cs sequences in Brassica Database (Figure 1). ABI1 had five homologous genes located in A01, A03, B05, A08 and B03; $A B I 2$ had two homologous genes located in $\mathrm{B} 02$ and A10; $A H G 1$ had two homologous genes located in A03 and B08; AHG3 had three homologous genes located in A05, B07 and B01; HAB1 and HAB2 both had one homologous gene located in the same chromosome B03; HAIl had two homologous genes located in A10 and B02; HAI2 had three homologous genes located in A09, B06 and A10 (Figure 1, Table 1). The comparable homologous gene numbers in A sub-genome and B sub-genome suggested tuber mustard genome experienced co-linearity gene duplication. However, the homologous genes of $H A B 1, H A B 2$ and $H A I 3$ were not duplicated or lost in tuber mustard, indicating that these genes existed a functional redundancy or divarication during the evolutionary process. The losses of genes during the genome duplication events also frequently exist in tuber mustard and other Brassica species, such as BjuPYLs family genes in tuber mustard [32] and chitinase family genes in B. rapa [33]. The promoters of clade A BjuPP2Cs family genes contained various of cis-acting elements, such as ABRE, GT1GMSCAM4, ARFAT and MYB1AT, which regulated plant response to abiotic and biotic stresses and hormone, indicating that the expression level of clade A BjuPP2Cs were regulated by hormone, abiotic and biotic stresses (Figure 4). Further we analyzed the expression pattern of clade A BjuPP2Cs under pathogen P. brassicae. The result showed that BjuABI1-5, BjuAHG1-1, BjuAHG3-2, BjuAHG3-3, BjuHAB1, BjuHAB2, BjuHAI1-1, BjuHAI2-1, BjuHAI2-3 and BjuHAI3 were highly induced by P. brassicae, suggesting that these genes regulated tuber mustard response to $P$. brassicae to mediate the formation of clubroot (Figure 7). The stem swollen of tuber mustard were divided into five stages, named D1 (the stems of 1-month-old seedlings, six leaf stage), D2 (the 
stems of 2-month-old seedlings, primary stage of stem swelling), D3 (the stems of 3-month-old seedlings, early stage of stem swelling), D4 (the stems of 4.5-month-old seedlings, fast-growing stage of stem swelling) and D5 (the stems of 5-month-old seedlings, last stage of stem swelling). In this study, we found that BjuABI1-1, BjuAHG1-1, BjuHAI2-2, BjuHAI2-3 and BjuHAI3 were inhibited in D2 to D4, in contrast, BjuABI1-3, BjuABI2-1, BjuHAB1 and BjuHAI1-1 were induced in D2 to D4 (Figure 8). The result suggested that clade A BjuPP2Cs might play roles in stem swelling of tuber mustard.

Gene duplication contained three evolutionary fates: subfunctionalization, neofunctionalization, or nonfunctionalization $[14,34]$. In our study, each of clade A BjuPP2Cs contained 1-5 copies with different gene structures and expression patterns. For example, BjuABI1-1, BjuABI1-2 and BjuABI1-3 contained 4 exons which is in accordance with AtABII, whereas, BjuABI1-4 and BjuABII-5 only contained 3 exons (Figure 2). The Serine/threonine phosphatases domain was all existed in BjuABI1s proteins (Figure 3). However, the expression patterns of BjuABIIs were significantly different, such as all of BjuABI1s except BjuABI1-5 were induced by ABA and only BjuABI1-2, BjuABI1-3 and BjuABI1-4 were induced by low temperature (Figure 6).

Gene expression analysis showed differences in clade A BjuPP2C genes expression pattern in tuber mustard. Our results indicated that clade A PP2C-based stem swollen regulation and $P$. brassicae response in tuber mustard has evolved distinctly. Different expression patterns of clade A BjuPP2C genes to $\mathrm{NaCl}$ stress, low temperature and ABA treatment indicated low conservation of gene expression patterns and functional divergence between $B$. juncea var. tumida and A. thaliana homologous genes.

\section{Conclusions}

Taken together, 20 clade A BjuPP2Cs were identified in tuber mustard and the transcript levels of clade A BjuPP2Cs under pathogen treatment, abiotic stresses treatment and different development stages were analyzed. The results indicated that 
the clade A BjuPP2Cs might play crucial role in regulating the formation of clubroot, swollen stem and response to abiotic stresses.

Author contribution statement $\mathrm{CL}$ and $\mathrm{CC}$ conceived the project and designed the experiments. YZ, LY, LC, LW, ZC, GC and RS performed the experiments and analyzed the data. CL and CC prepared the manuscript. CC and CL revised the manuscript. All authors read and approved the final manuscript.

Funding: This work was supported by the Chongqing Basic Research and Frontier Exploration Project (Program No. cstc2018jcyjAX0628 and cstc2020jcyj-msxmX0770), the Science and Technology Research Program of Chongqing Municipal Education Commission (Grant No. KJQN201801434 and Grant No. KJQN201901438) and the Open Project of the Chongqing key Laboratory for Conservation and Utilization of Characteristic Plant Resources in Wuling Mountain Area (Grant No. TEZWKFKT202002).

\section{Declarations}

Conflicts of interests The authors declare that they have no competing interests for this research.

Data availability statements All data generated or analysed during this study are included in this published article and its supplementary information files.

\section{References}

1. Cao J, Jiang M, Li P, Chu Z. Genome-wide identification and evolutionary analyses of the PP2C gene family with their expression profiling in response to multiple 
stresses in Brachypodium distachyon. BMC Genomics. 2016, 17: 175.

2. Xue T, Wang D, Zhang S, Ehlting J, Ni F, Jakab S, Zheng C, Zhong Y. Genome-wide and expression analysis of protein phosphatase $2 \mathrm{C}$ in rice and Arabidopsis. BMC genomics. 2008, 9: 550.

3. Cohen P. The structure and regulation of protein phosphatases. Annu Rev Biochem. 1989, 58: 453-508.

4. Finkelstein R R, Gampala S S, Rock C D. Abscisic acid signaling in seeds and seedlings. Plant Cell. 2002, 14: S15-45.

5. Koornneef M, Hanhart C J, Hilhorst H W, Karssen C M. In vivo inhibition of seed development and reserve protein accumulation in recombinants of abscisic acid biosynthesis and responsiveness mutants in Arabidopsis thaliana. Plant Physiol. 1989, 90: 463-469.

6. Kong L, Cheng J, Zhu Y, Ding Y, Meng J, Chen Z, Xie Q, Guo Y, Li J, Yang S, Gong Z. Degradation of the ABA co-receptor ABI1 by PUB12/13 U-box E3 ligases. Nat Commun. 2015, 6: 8630.

7. Fujii H, Chinnusamy V, Rodrigues A, Rubio S, Antoni R, Park S Y, Cutler S R, Sheen J, Rodriguez P L, Zhu J K. In vitro reconstitution of an abscisic acid signalling pathway. Nature. 2009, 462: 660-664.

8. Ma Y, Szostkiewicz I, Korte A, Moes D, Yang Y, Christmann A, Grill E. Regulators of PP2C phosphatase activity function as abscisic acid sensors. Science. 2009, 324: 1064-1068.

9. Park S Y, Fung P, Nishimura N, Jensen D R, Fujii H, Zhao Y, Lumba S, Santiago J, Rodrigues A, Chow T F, Alfred S E, Bonetta D, Finkelstein R, Provart N J, Desveaux D, Rodriguez P L, McCourt P, Zhu J K, Schroeder J I, Volkman B F, Cutler S R. Abscisic acid inhibits type $2 \mathrm{C}$ protein phosphatases via the PYR/PYL family of START proteins. Science. 2009, 324: 1068-1071.

10. Merlot S, Gosti F, Guerrier D, Vavasseur A, Giraudat J. The ABI1 and ABI2 protein phosphatases $2 \mathrm{C}$ act in a negative feedback regulatory loop of the abscisic acid signaling pathway. Plant J. 2001, 25: 295-303.

11. Nishimura N, Yoshida T, Kitahata N, Asami T, Shinozaki K, Hirayama T. ABA-Hypersensitive Germination1 encodes a protein phosphatase 2C, an 
essential component of abscisic acid signaling in Arabidopsis seed. Plant J. 2007, 50(6): 935-949.

12. Carrillo-Barral N, Rodríguez-Gacio M D C, Matilla A J. Delay of Germination-1 (DOG1): A key to understanding seed dormancy. Plants (Basel). 2020, 9(4): 480.

13. Wang Z, Ji H, Yuan B, Wang S F, Su C, Yao B, Zhao H T, Li X. ABA signalling is fine-tuned by antagonistic HAB1 variants. Nat Commun. 2015, 6: 8138 .

14. Khan N, Ke H, Hu CM, Naseri E, Haider M S, Ayaz A, Amjad Khan W, Wang J, Hou X. Genome-wide identification, evolution, and transcriptional profiling of PP2C Gene family in Brassica rapa. Biomed Res Int. 2019, 15.

15. Ludwikik A, Babula-Skowrońska D, Szczepaniak M, Belter N, Dominiak E, Sadowski J. Expression profiles and genomic organisation of group a protein phosphatase 2c genes in Brassica oleracea. Annals of Applied Biology. 2013, 163(1): 124-134.

16. Yang J, Liu D, Wang X, Ji C, Cheng F, Liu B, Hu Z, Chen S, Pental D, Ju Y, Yao P, Li X, Xie K, Zhang J, Wang J, Liu F, Ma W, Shopan J, Zheng H, Mackenzie S A, Zhang M. The genome sequence of allopolyploid Brassica juncea and analysis of differential homoeolog gene expression influencing selection. Nat Genet. 2016, 48: 1225-1232.

17. Shi H, Wang L L, Sun L T, Dong L L, Liu B, Chen L P. Cell division and endoreduplication play important roles in stem swelling of tuber mustard (Brassica juncea Coss. var. tumida Tsen et Lee). Plant Biol. 2012, 14: 956-963.

18. Wang D, Sun T, Zhao S, Pan L, Liu H, Tian X. Physiological change alters endophytic bacterial community in clubroot of tumorous stem mustard infected by Plasmodiophora brassicae. BMC Microbiol. 2020, 20(1): 244.

19. Bork P, Brown N P, Hegyi H, Schultz J. The protein phosphatase 2C (PP2C) superfamily: detection of bacterial homologues. Protein Sci. 1996, 5(7): 1421-1425.

20. Nag R, Maity M K, Dasgupta M. Dual DNA binding property of ABA insensitive 3 like factors targeted to promoters responsive to ABA and auxin. Plant Mol 
Biol. 2005, 59: 821-838.

21. Redman J, Whitcraft J, Johnson C, Arias J. Abiotic and biotic stress differentially stimulate as-1 element activity in Arabidopsis. Plant Cell Rep. 2002, 21: 180-185.

22. Nakashima K, Fujita Y, Katsura K, Maruyama K, Narusaka Y, Seki M, Shinozaki K, Yamaguchi-Shinozaki K. Transcriptional regulation of ABI3- and ABA-responsive genes including $R D 29 B$ and $R D 29 A$ in seeds, germinating embryos, and seedlings of Arabidopsis. Plant Mol Biol. 2006, 60: 51-68.

23. Abe H, Urao T, Ito T, Seki M, Shinozaki K, Yamaguchi-Shinozaki K. Arabidopsis AtMYC2 (bHLH) and AtMYB2 (MYB) function as transcriptional activators in abscisic acid signaling. Plant Cell. 2003, 15: 63-78.

24. Abe H, Yamaguchi-Shinozaki k, Urao T, Iwasaki T, Hosokawa D, Shinozaki K. Role of Arabidopsis MYC and MYB homologs in drought- and abscisic acid-regulated gene expression. Plant Cell. 1997, 9: 1859-1868.

25. Xue G P. Characterisation of the DNA-binding profile of barley HvCBF1 using an enzymatic method for rapid, quantitative and high-throughput analysis of the DNA-binding activity. Nucleic Acids Res. 2002, 30(15): e77.

26. Xue G P. The DNA-binding activity of an AP2 transcriptional activator HvCBF2 involved in regulation of low-temperature responsive genes in barley is modulated by temperature. Plant J. 2003, 33: 373-383.

27. Baker S S, Wilhelm K S, Thomashow M F. The 5'-region of Arabidopsis thaliana cor15a has cis-acting elements that confer cold-, drought- and ABA-regulated gene expression. Plant Mol Biol. 1994, 24: 701-713.

28. Park H C, Kim M L, Kang Y H, Jeon J M, Yoo J H, Kim M C, Park C Y, Jeong J C, Moon B C, Lee J H, Yoon H W, Lee S H, Chung W S, Lim C O, Lee S Y, Hong J C, Cho M J. Pathogen- and NaCl-induced expression of the SCaM-4 promoter is mediated in part by a GT-1 box that interacts with a GT-1-like transcription factor. Plant Physiol. 2004, 135: 2150-2161.

29. Brown R L, Kazan K, McGrath K C, Maclean D J, Manners J M. A role for the GCC-box in jasmonate-mediated activation of the PDF1.2 gene of Arabidopsis. 
Plant Physiol. 2003, 132: 1020-1032.

30. Chakravarthy S. The tomato transcription factor Pti4 regulates defense-related gene expression via GCC box and non-GCC box cis elements. Plant Cell. 2003, 15: 3033-3050.

31. Cai Z, Zeng DE, Liao J, Cheng C, Sahito Z A, Xiang M, Fu M, Chen Y, Wang D. Genome-wide analysis of auxin receptor family genes in Brassica juncea var. tumida. Genes. 2019, 10: 165.

32. Cheng C, Zhong Y, Cai Z, Su R, Li C. Genome-wide identification and gene expression analysis of ABA receptor family genes in Brassica juncea var. tumida. Genes (Basel). 2019, 10(6): 470.

33. Chen J, Piao Y, Liu Y, Li X, Piao Z. Genome-wide identification and expression analysis of chitinase gene family in Brassica rapa reveals its role in clubroot resistance. Plant Sci. 2018, 270: 257-267.

34. Lynch M, Conery J S. The evolutionary fate and consequences of duplicate genes. Science. 2000, 290(5494): 1151-11555.

\section{Figure legends}

Table 1 The clade A BjuPP2Cs family members in B. juncea var. tumida.

Figure 1. The distribution of clade A BjuPP2Cs in B. juncea var. tumida chromosomes. Twenty identified clade A BjuPP2Cs were mapped to the 13 of 18 chromosomes. The chromosome name is at the top of each bar. The scale of the chromosome is in millions of bases $(\mathrm{Mb})$.

Figure 2. The phylogenic tree and gene structures of clade A PP2C family genes. The phylogenic tree was built using the neighbor-joining (NJ) method and the exon-intron structure of clade A $P P 2 C$ homologs was drawn according to their phylogenic relationships. The blue boxes and black lines denoted exons and introns, respectively. 
Figure 3. The conserved motifs of clade A PP2C homologs proteins. These motifs were identified using the online software of SMART, and colored boxes indicated conserved motifs and black lines represent non-conserved sequences.

Figure 4. The promoter cis-elements analysis of clade A BjuPP2Cs. The $2 \mathrm{~kb}$ DNA fragments upstream of the ATG staring code of clade A BjuPP2Cs were analyzed using online analysis software PlantCARE and PLACE.

Figure 5. Expression patterns of clade A BjuPP2Cs in different tissues. Tissue specific expression pattern of clade A BjuPP2Cs were analyzed by qPCR. Data were normalized to the expression level of BjuActin3. The values are means \pm standard error. Three independent biological repeats were performed.

Figure 6. The expression patterns of clade A BjuPP2Cs under abiotic stresses. Total RNA was extracted from tuber mustard seedlings treated with $\mathrm{ABA}, \mathrm{NaCl}$ and $4^{\circ} \mathrm{C}$ for $3 \mathrm{~h}$. Data were normalized to the expression level of BjuActin3. The values are means \pm standard error. Three independent biological repeats were performed.

Figure 7. The expression patterns of clade A BjuPP2Cs under pathogen treatment. Total RNA was extracted from tuber mustard seedlings treated with $P$. Brassicae at the indicated time points. Data were normalized to the expression level of BjuActin3. The values are means \pm standard error. Three independent biological repeats were performed.

Figure 8. The expression patterns of clade A BjuPP2Cs at different stages of stem swelling. Total RNA was extracted from the seedlings of D1, D2, D3, D4 and D5 stages. Data were normalized to the expression level of BjuActin3. The values are means \pm standard error. Three independent biological repeats were performed. 
Table S1 The primers used in this study.

Fig. S1 Multiple alignment of conserved motifs of the clade A PP2C family proteins. The multiple alignment of conserved motifs was constructed using CLUSTALW. The clade A PP2C family proteins showed 11 conserved sequence motifs, named motif 1 to motif 11 along the top (blue font diaplay). Residues conserved of clade A PP2Cs highlighted in red (totally conservation) and yellow (high conservation). 


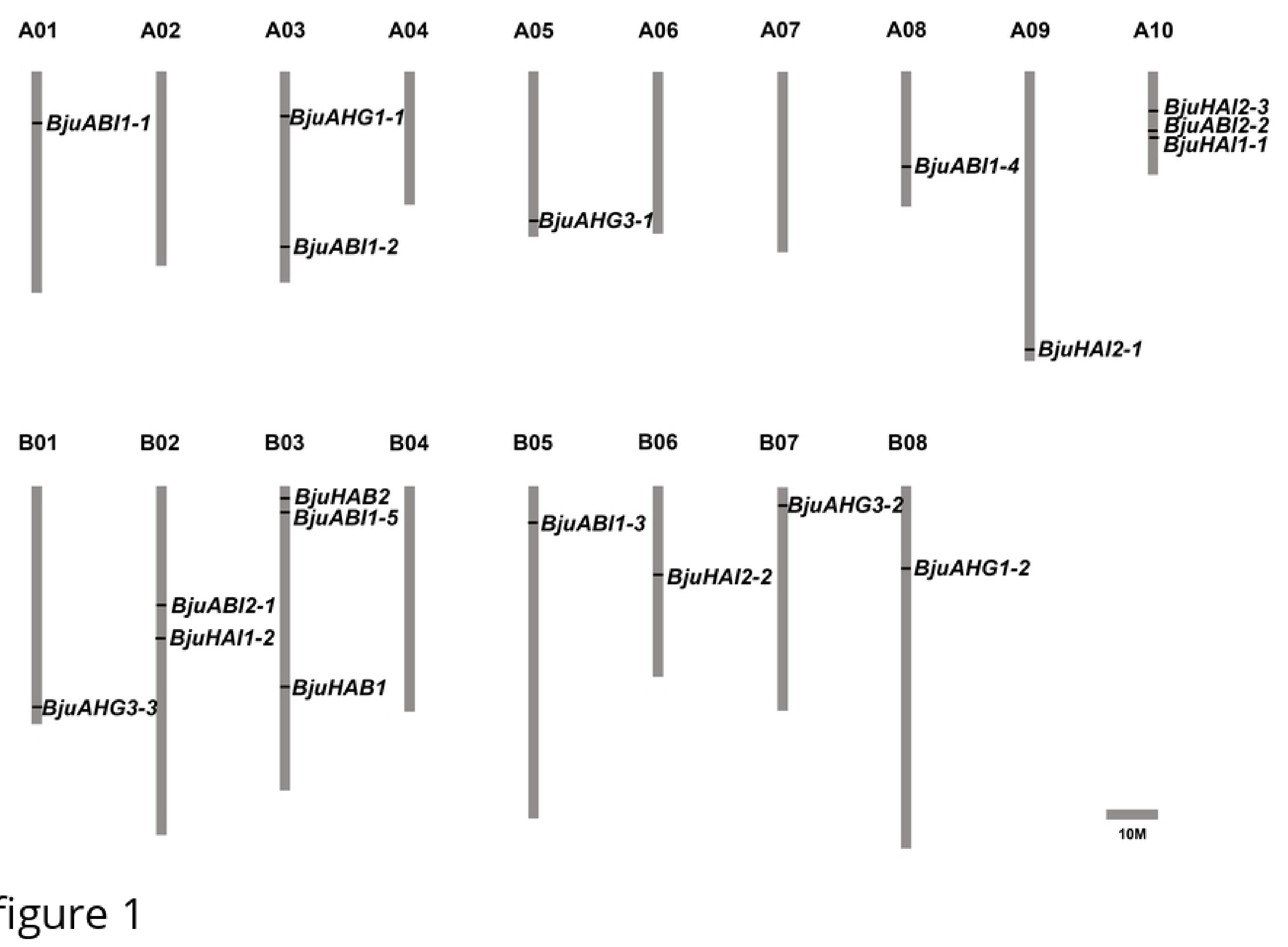




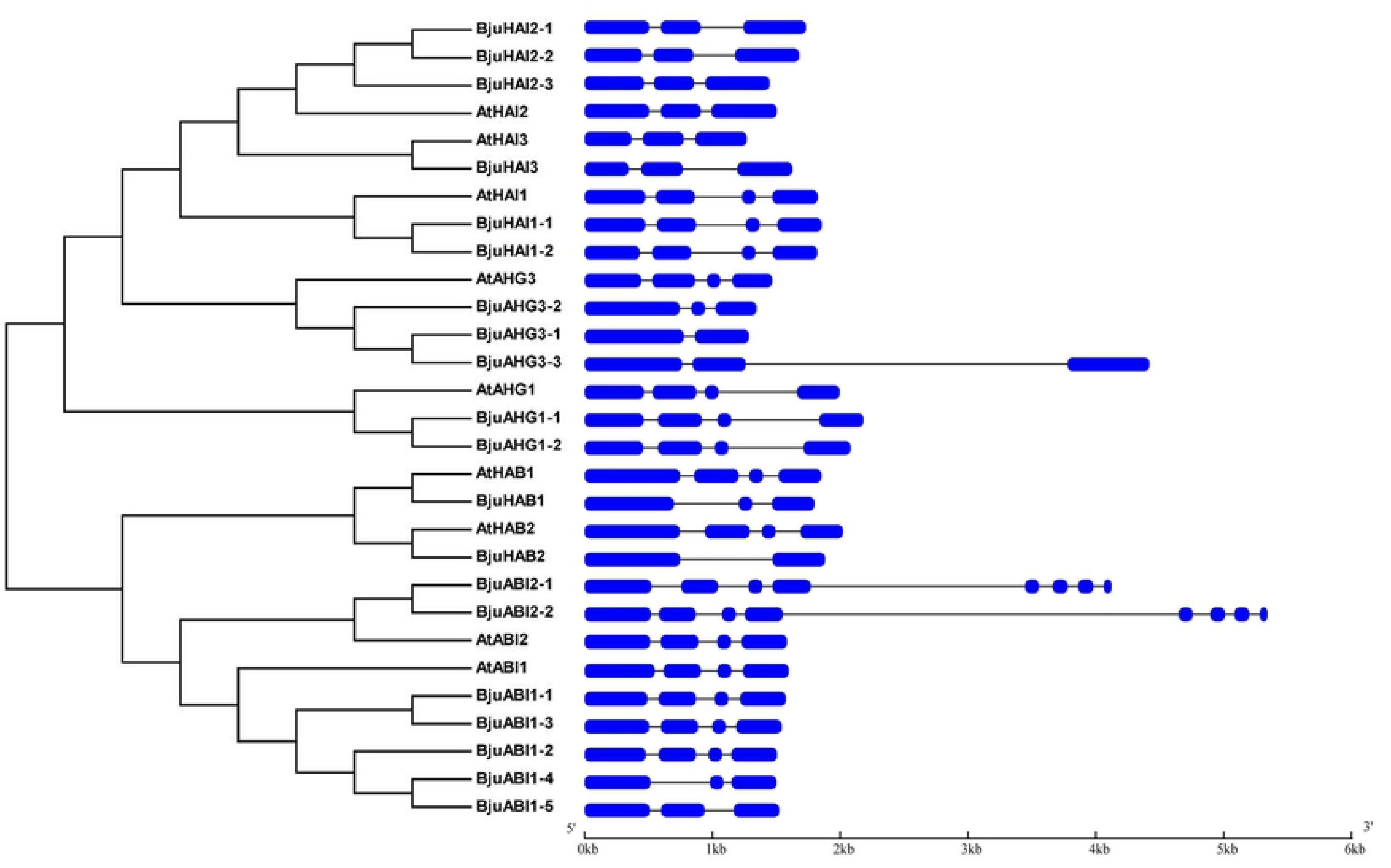

Legend:

Exon - Intron

figure 2 

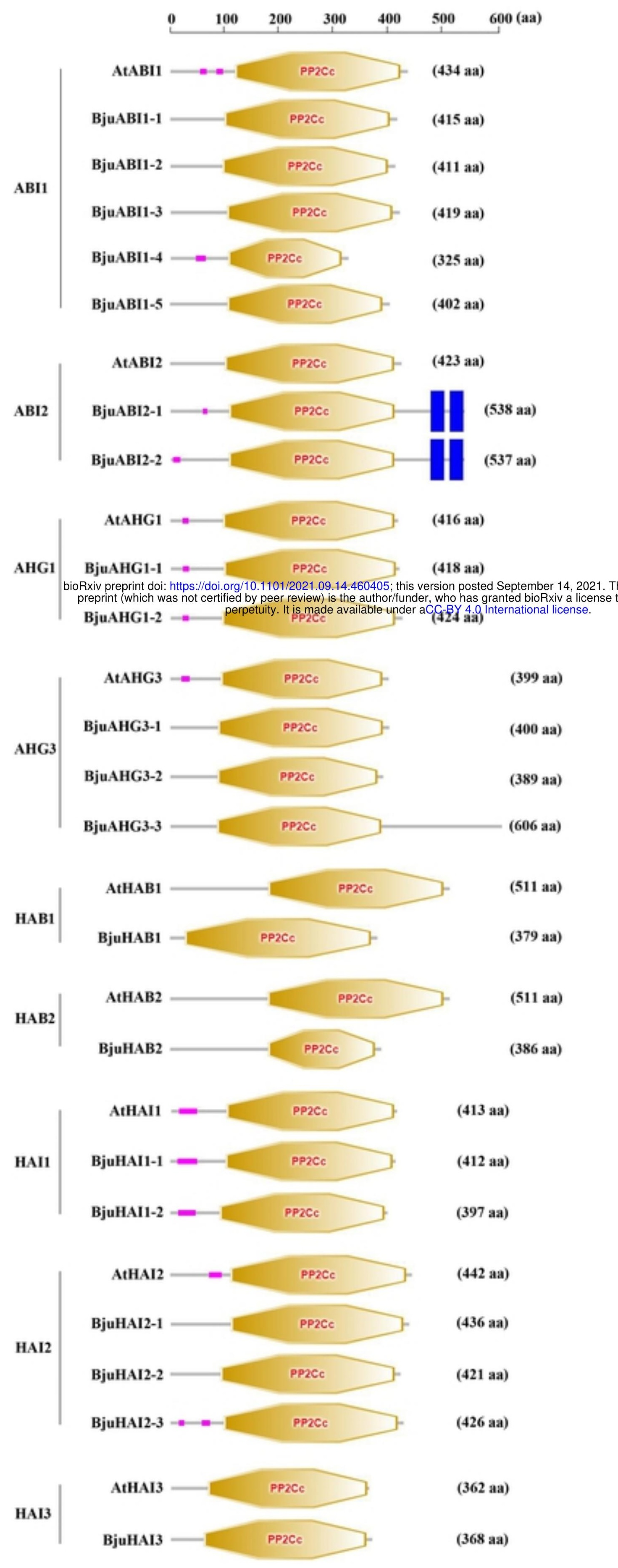

- Low complexity region Transmembrane region

\section{figure 3}




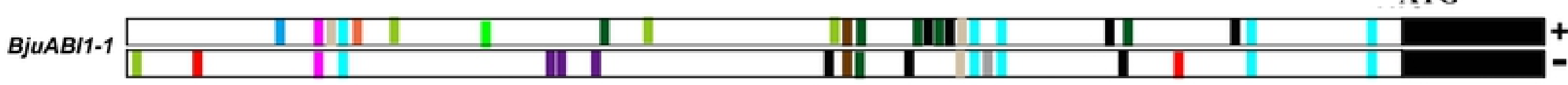

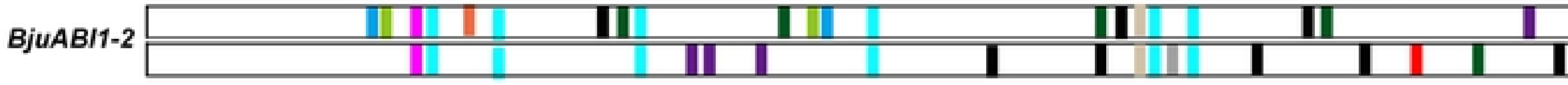

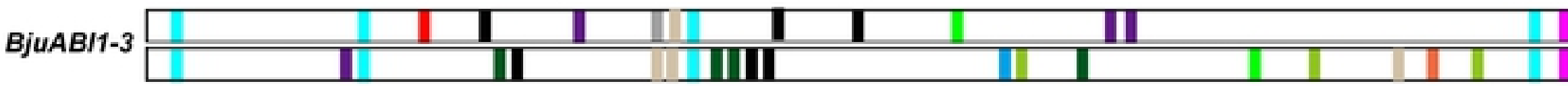

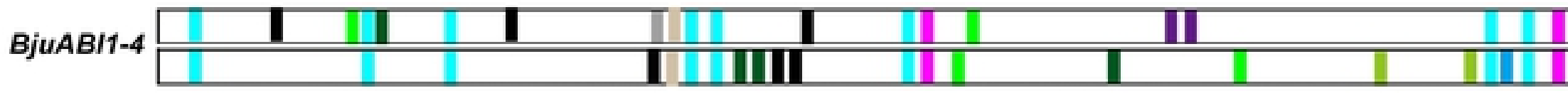

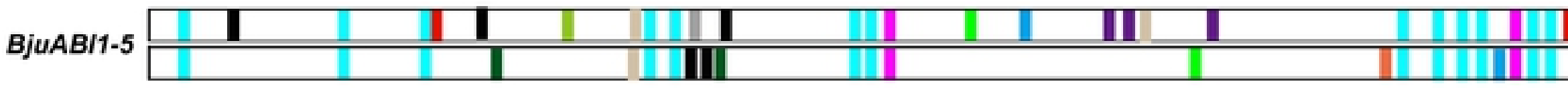

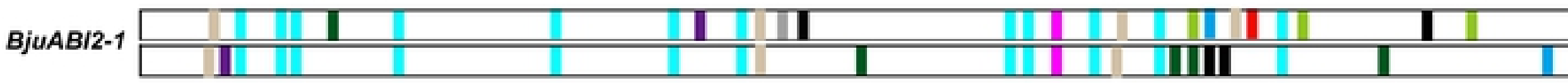

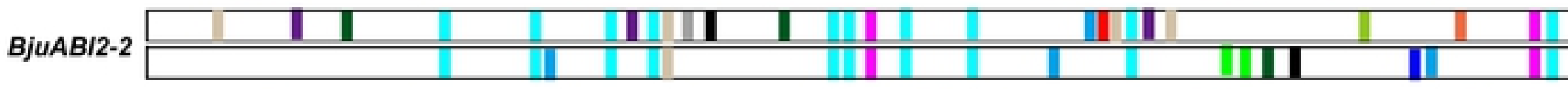

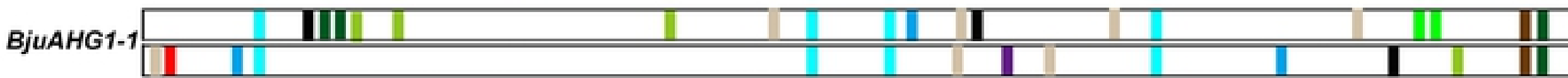

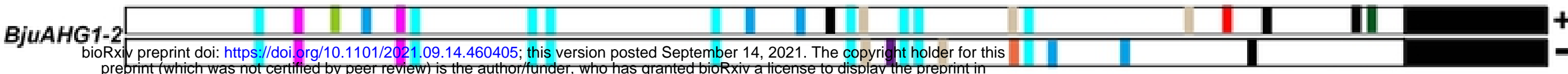

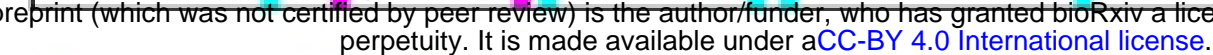

BjuAHG3-1

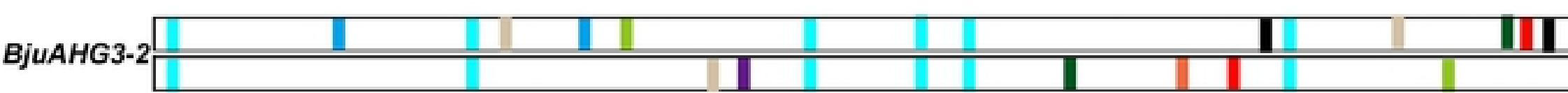

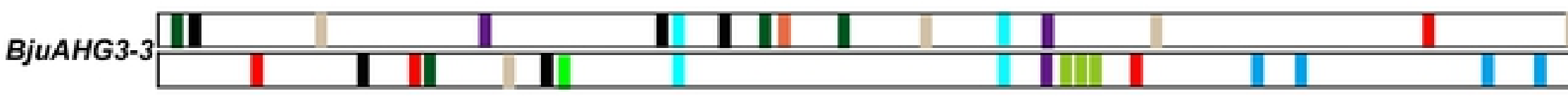
BjuHAB1 BjuHAB2

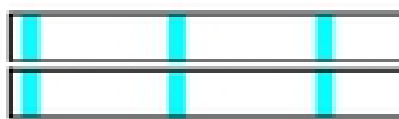

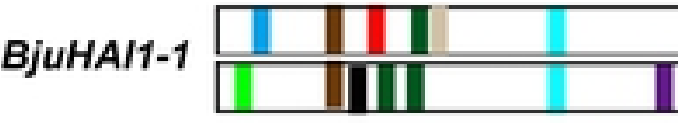
III प1

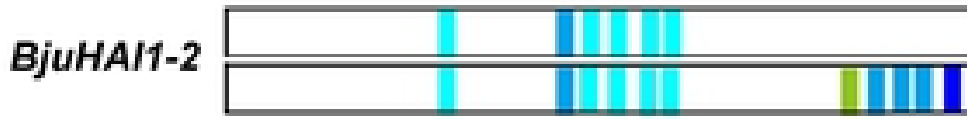

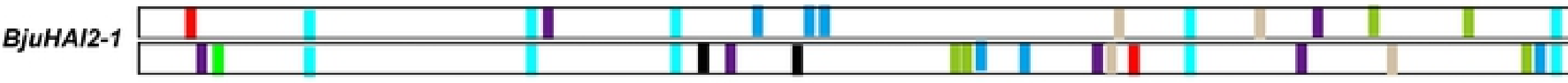

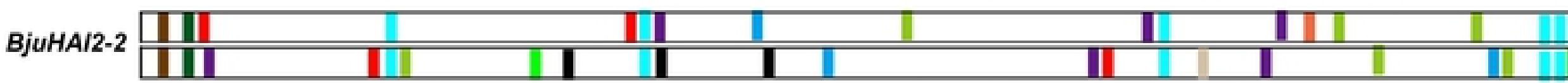

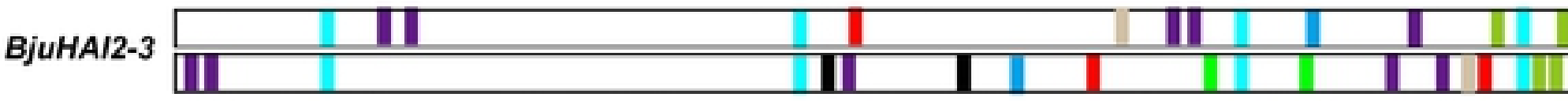

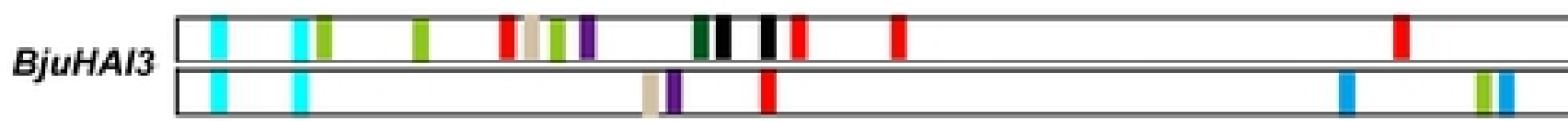

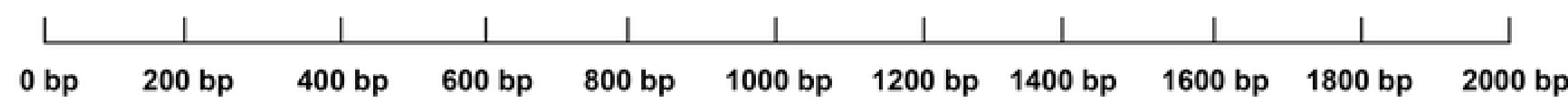

| CANNTG (responsive to dehydration stress) MYCCONSENSUSAT

】 CTAACCA (responsive to dehydration stress) MYBATRD22

| GCCGCC (responsive to pathogen) GCCCORE

I GTCGAC (responsive to low temperature) CRTDREHVCBF2

I TGTCTC (responsive to auxin) ARFAT

】 TGACG (responsive to auxin and salicylic acid) ASF1MOTIFCAMV

| RYACGTGGYR (responsive to Abscisic acid, ABA) ABREATRD22
WAACCA (responsive to dehydration stress) MYB1AT

I GAAAAA (responsive to pathogen and salt stress) GT1GMSCAM4

| GTTAGTT (responsive to defence) MYB1LEPR

I CCGAC (responsive to low temperature) LTRECOREATCOR15

I CATATG (responsive to auxin) CATATGgMSAUR

ACGTG (responsive to Abscisic acid, ABA) ABRE

I RYCGAC (responsive to dehydration stress) CBFHV 
A

B

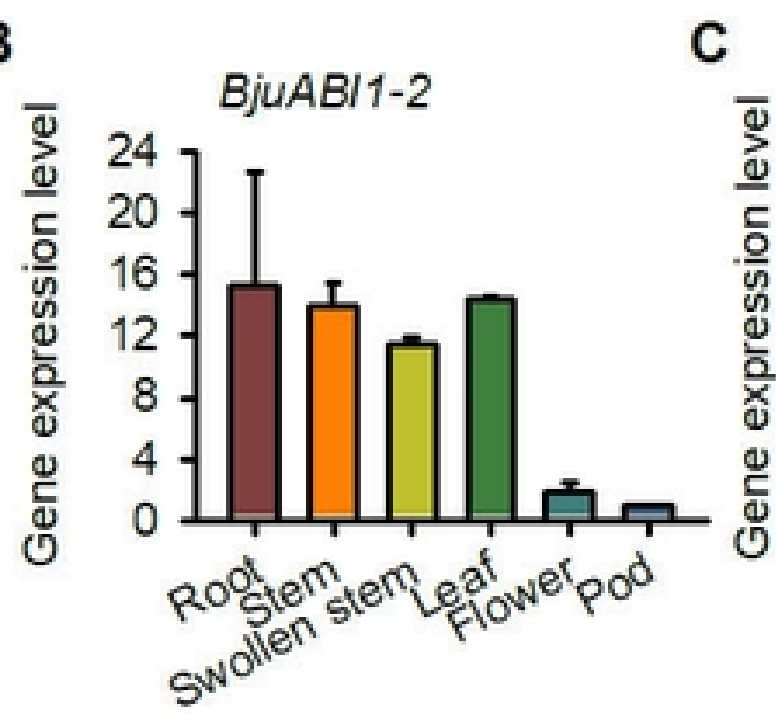

BjuABI1-3

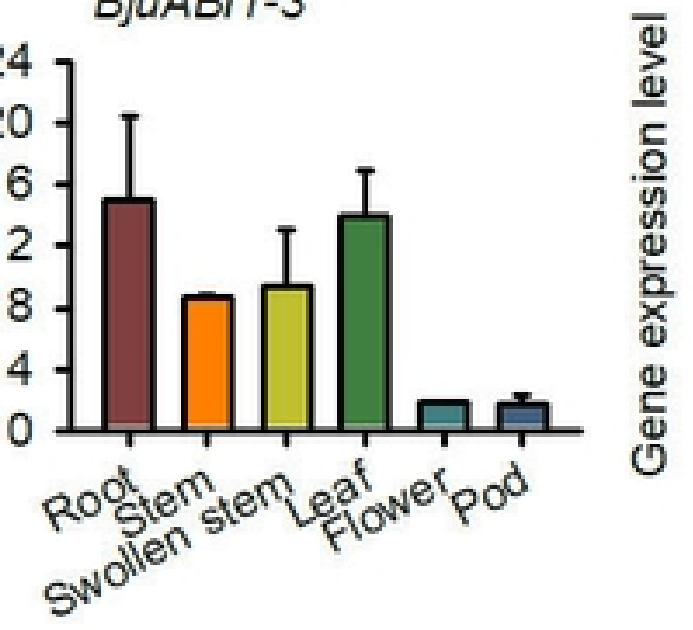

BjuABI1-4

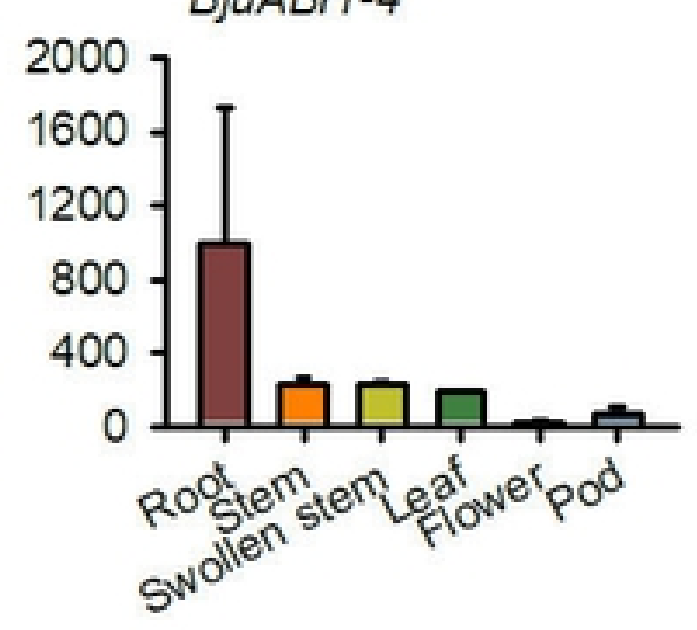

E

F

G

H
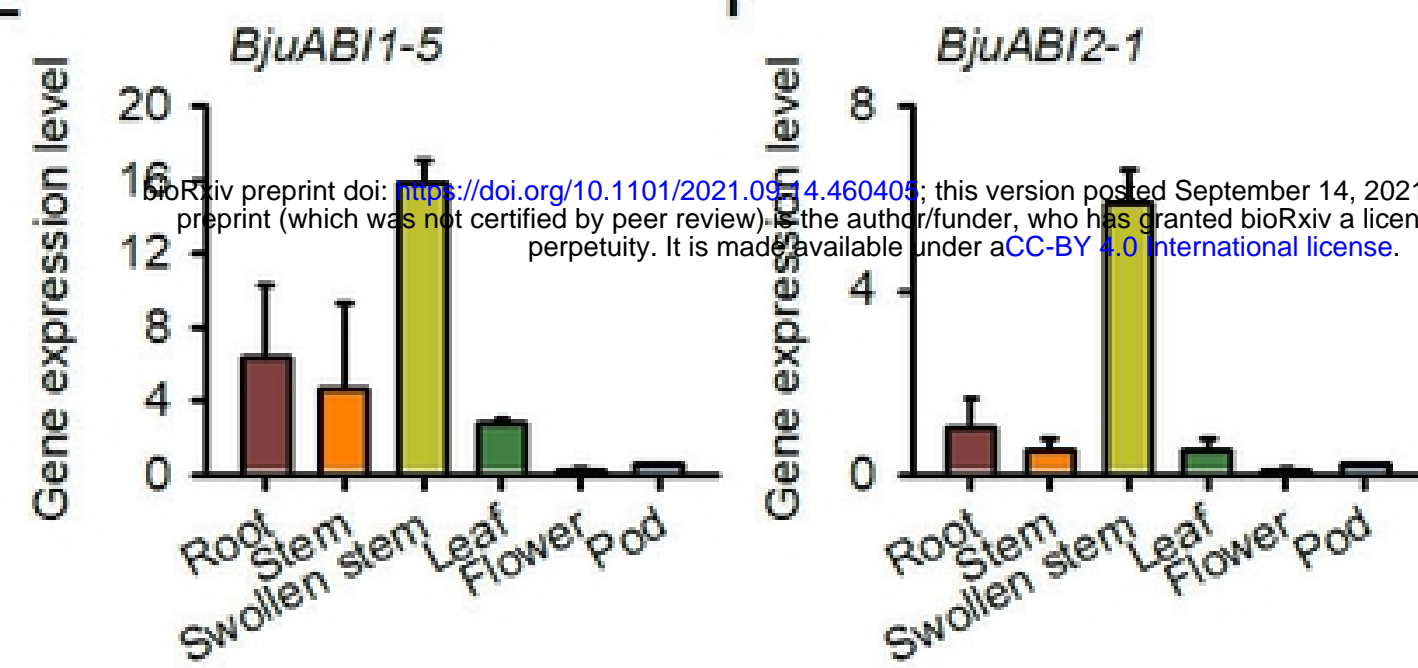

$B j u A B / 2-2$

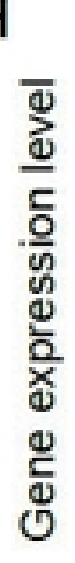

BjuAHG 1-1

I

J

K

SN L

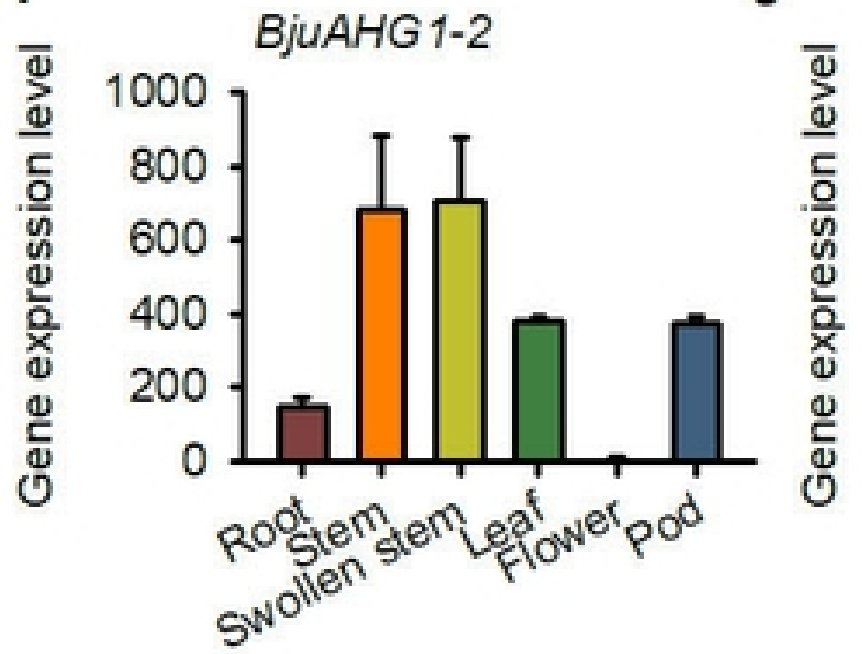

BjuAHG3-1
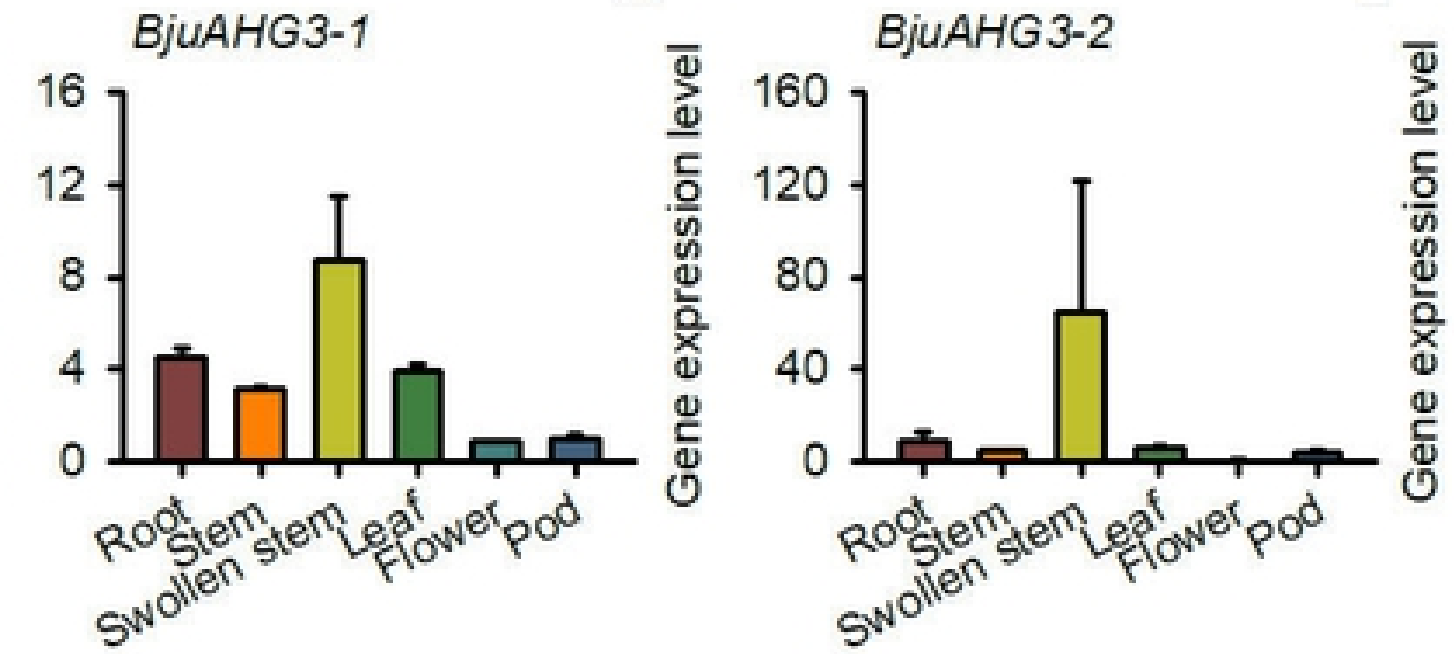

BjuAHG3-3

M

N

0

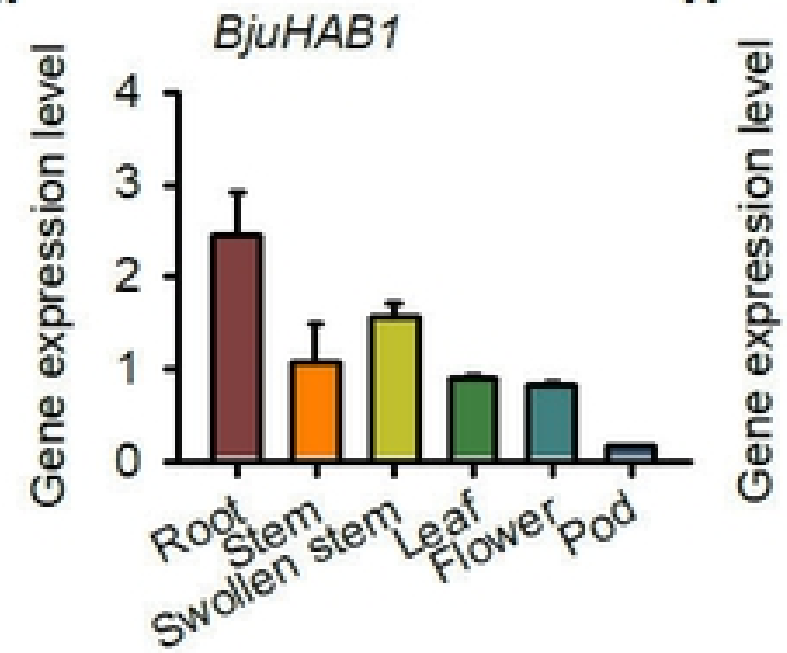

BjuHAB2

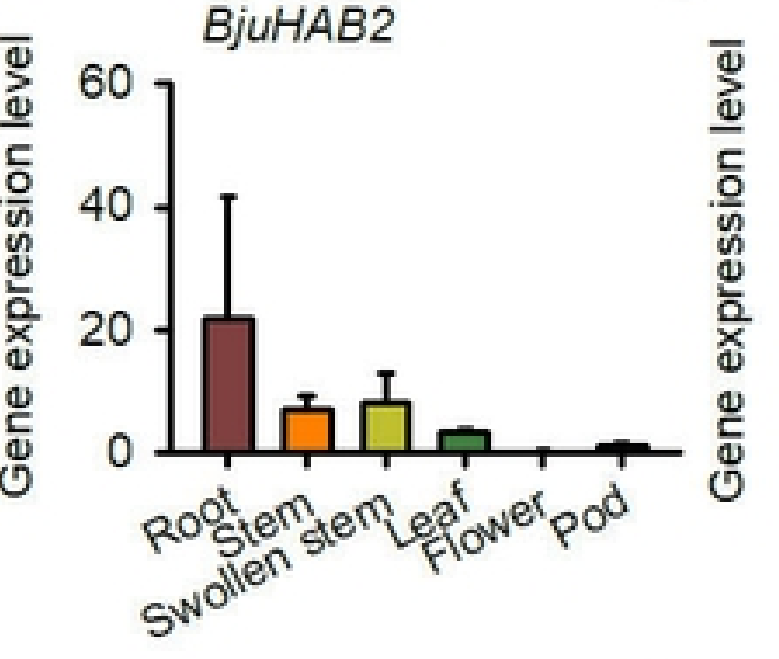

BjuHAl1-1 P

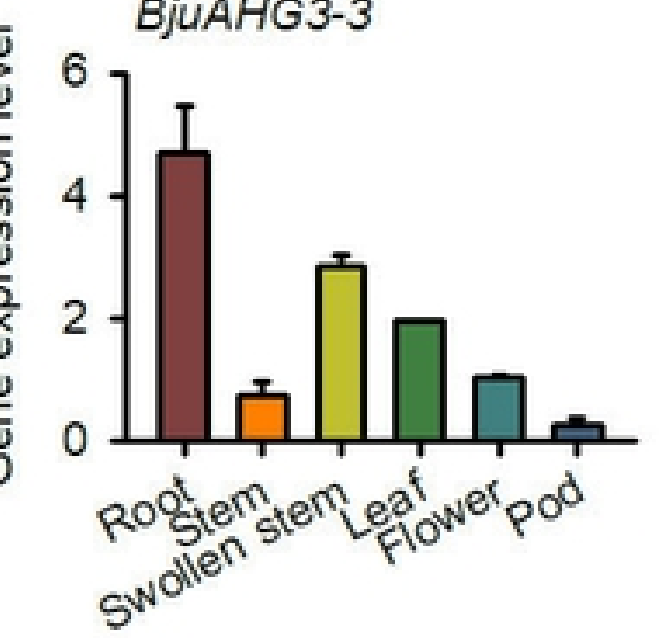

Q
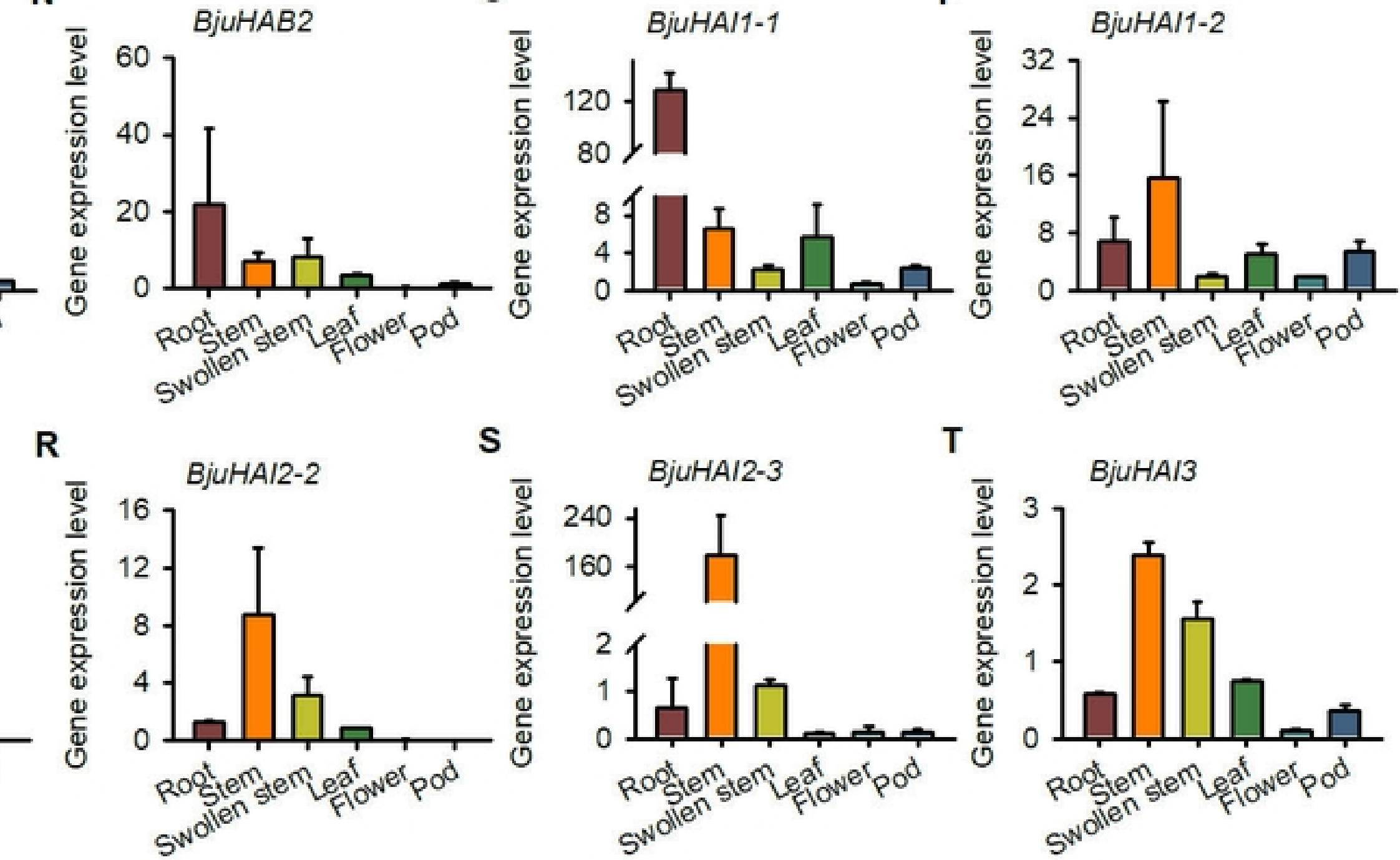

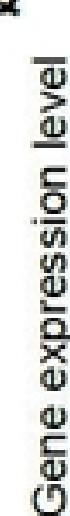

BjuHA/2-1

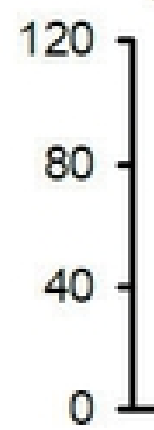

Roştermstem eqiowerpod
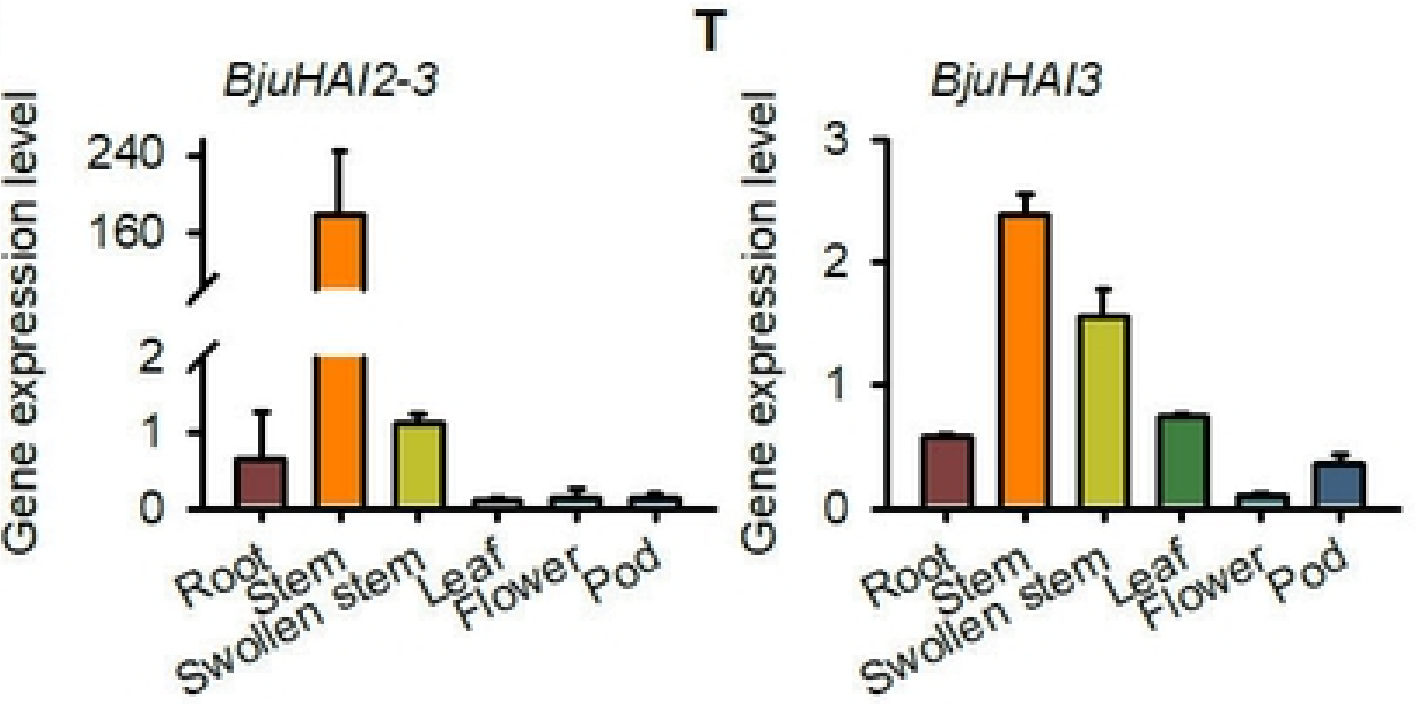

figure 5 
A BjuAB/1-1

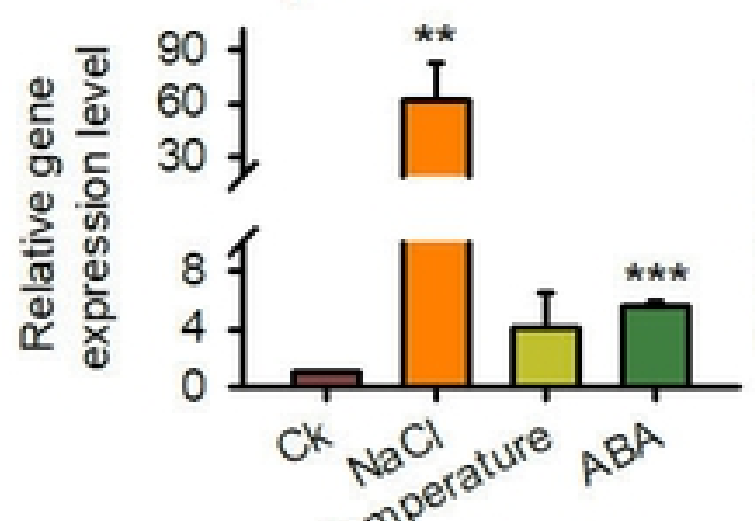

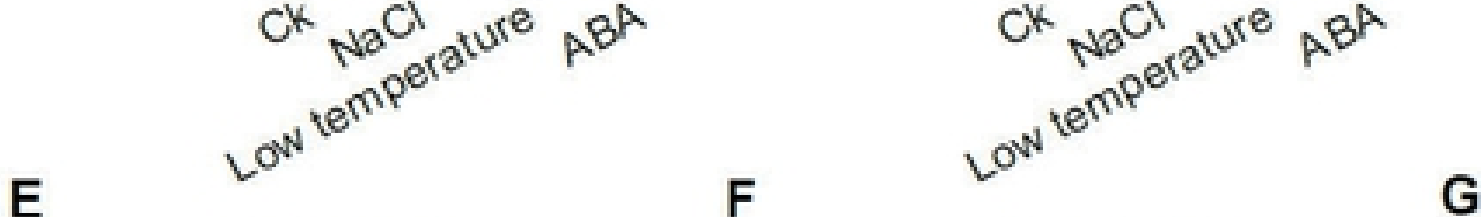
E BjuAB/1-5
B

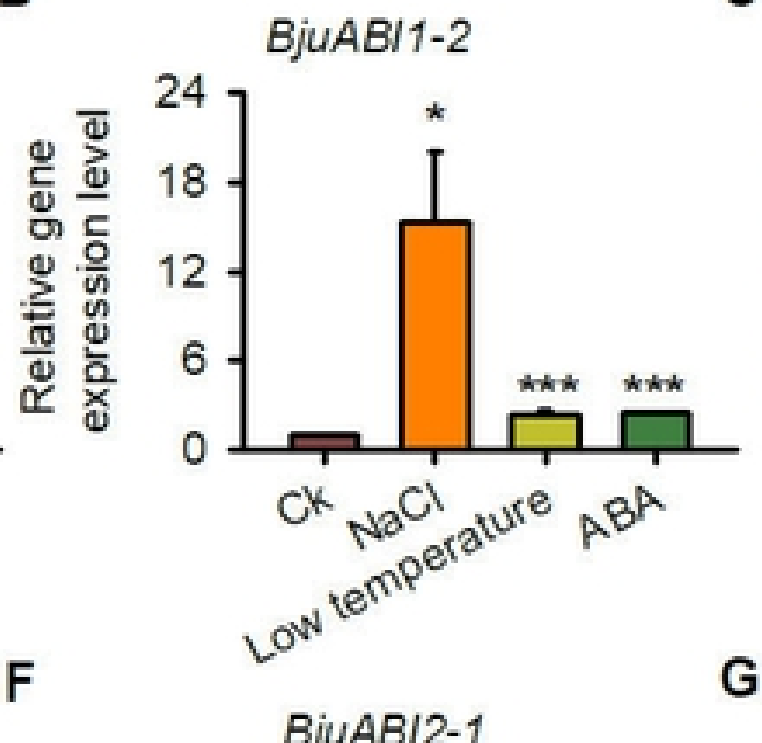

C

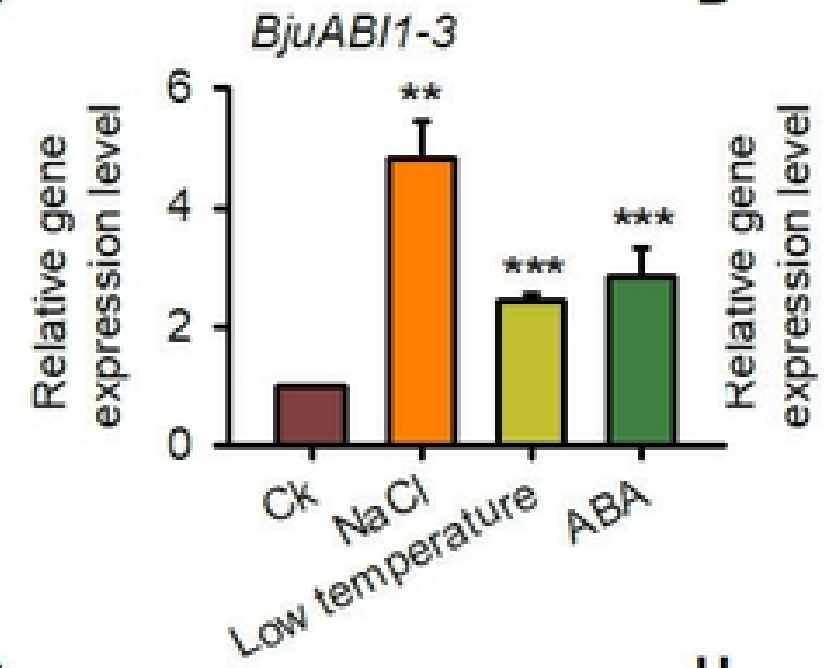

BjuAB/2-2

H
BjuAHG3-2

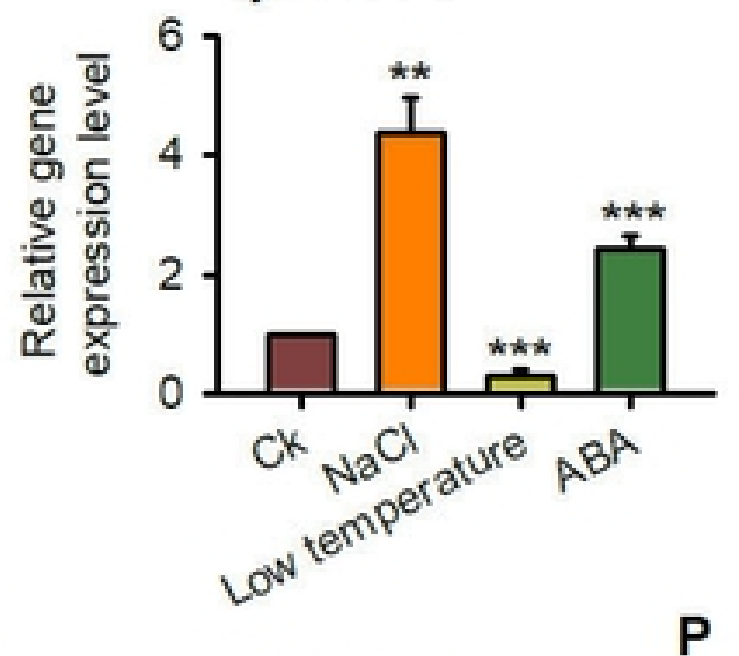

BjuHAl1-1

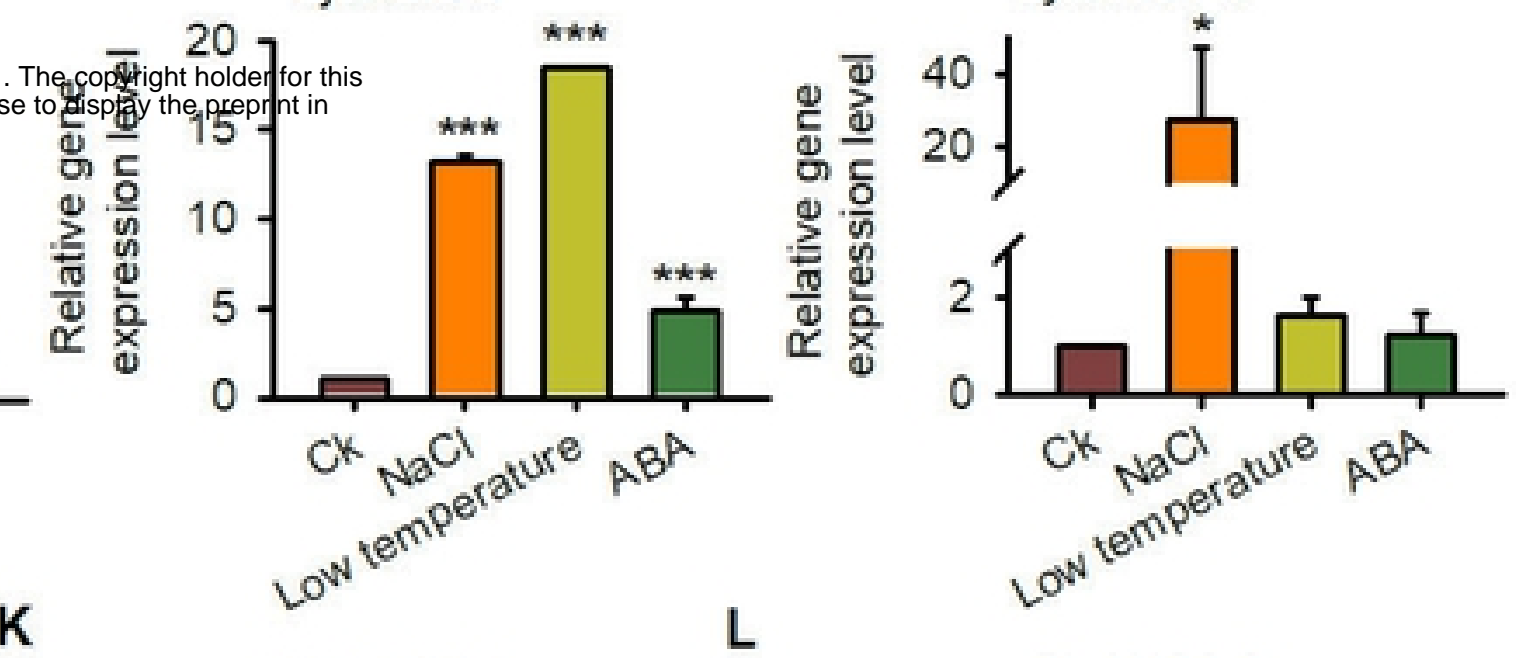

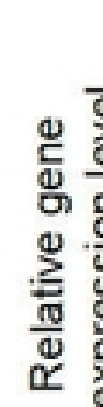

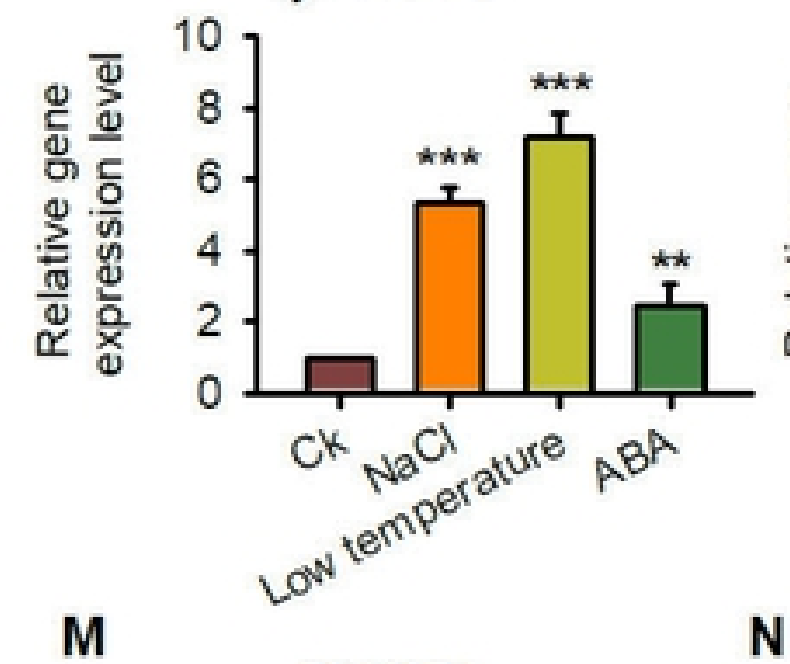

BjuHAB1
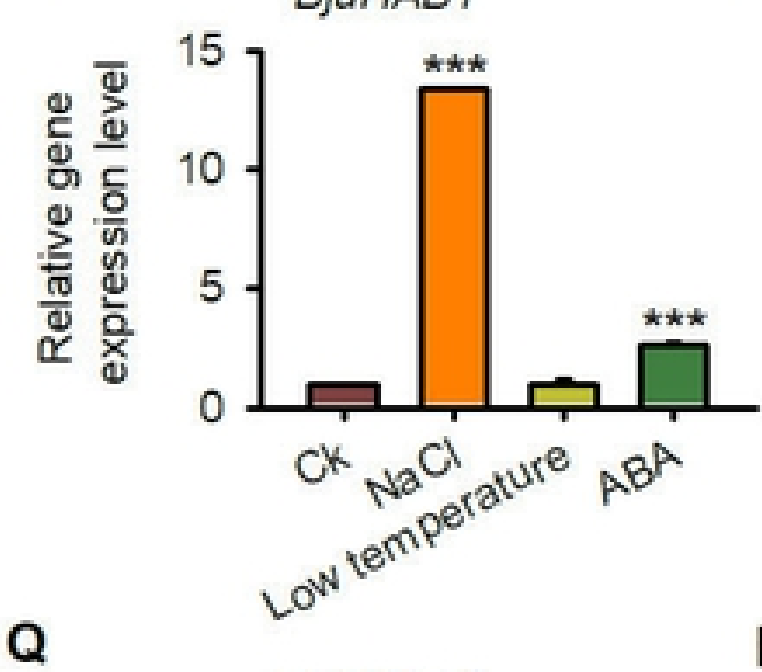

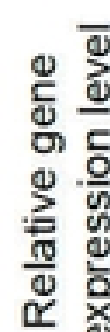

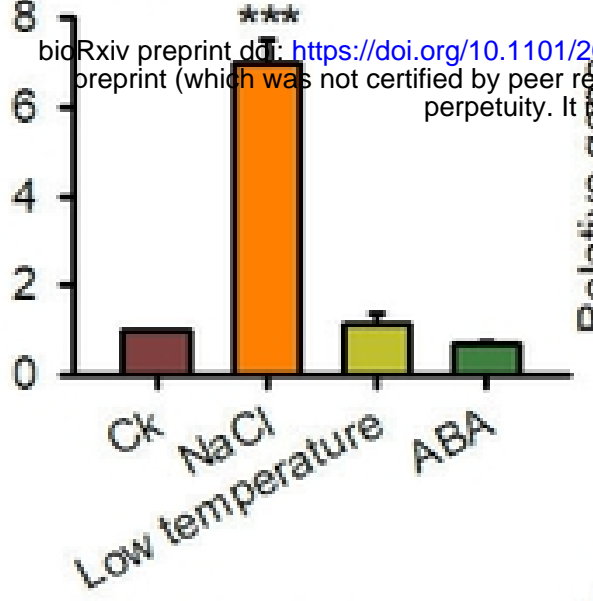

BjuHAI2-1

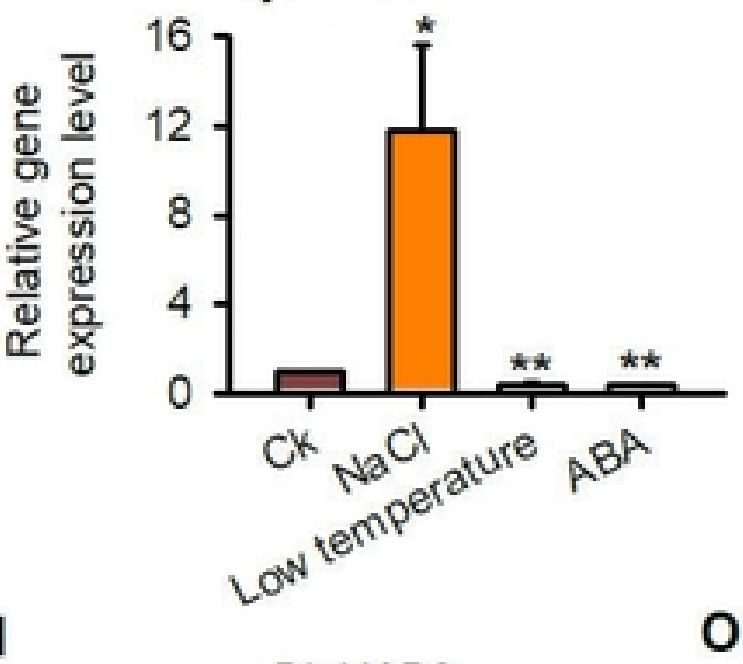

BjuHAB2

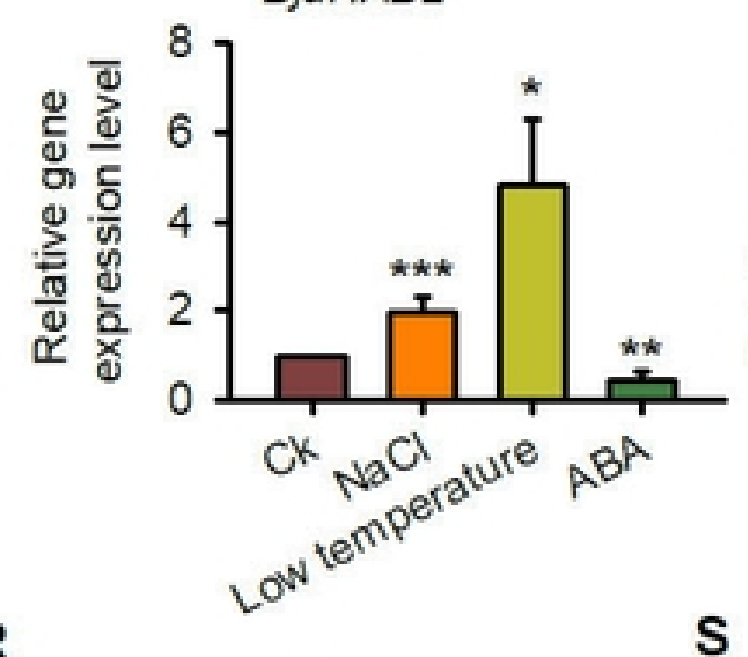

BjuHAI2-2

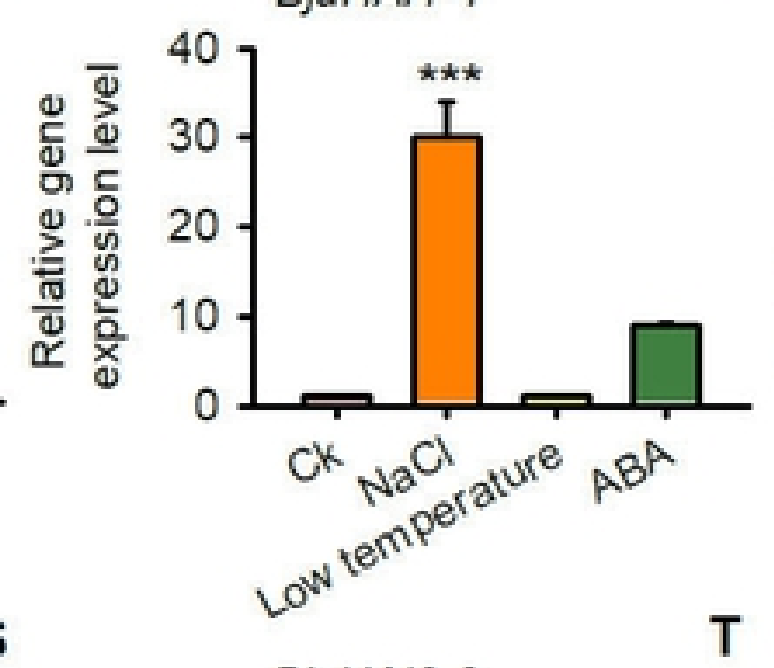

BjuHAI2-3

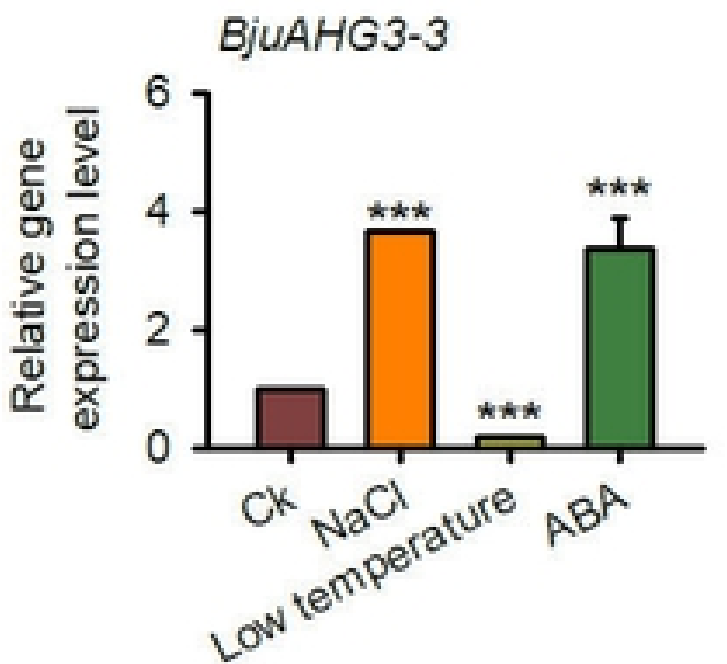

BjuHAl1-2
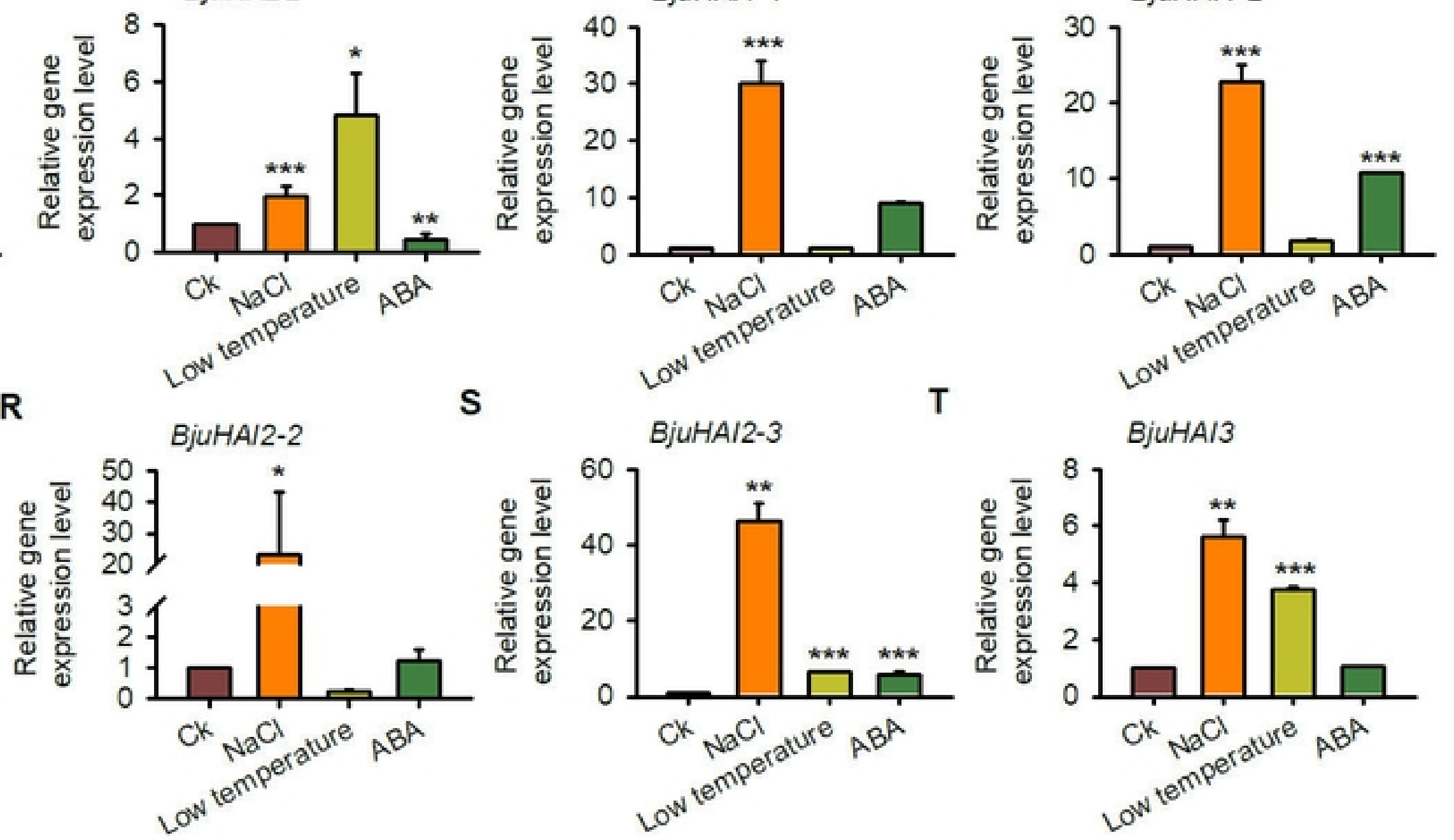


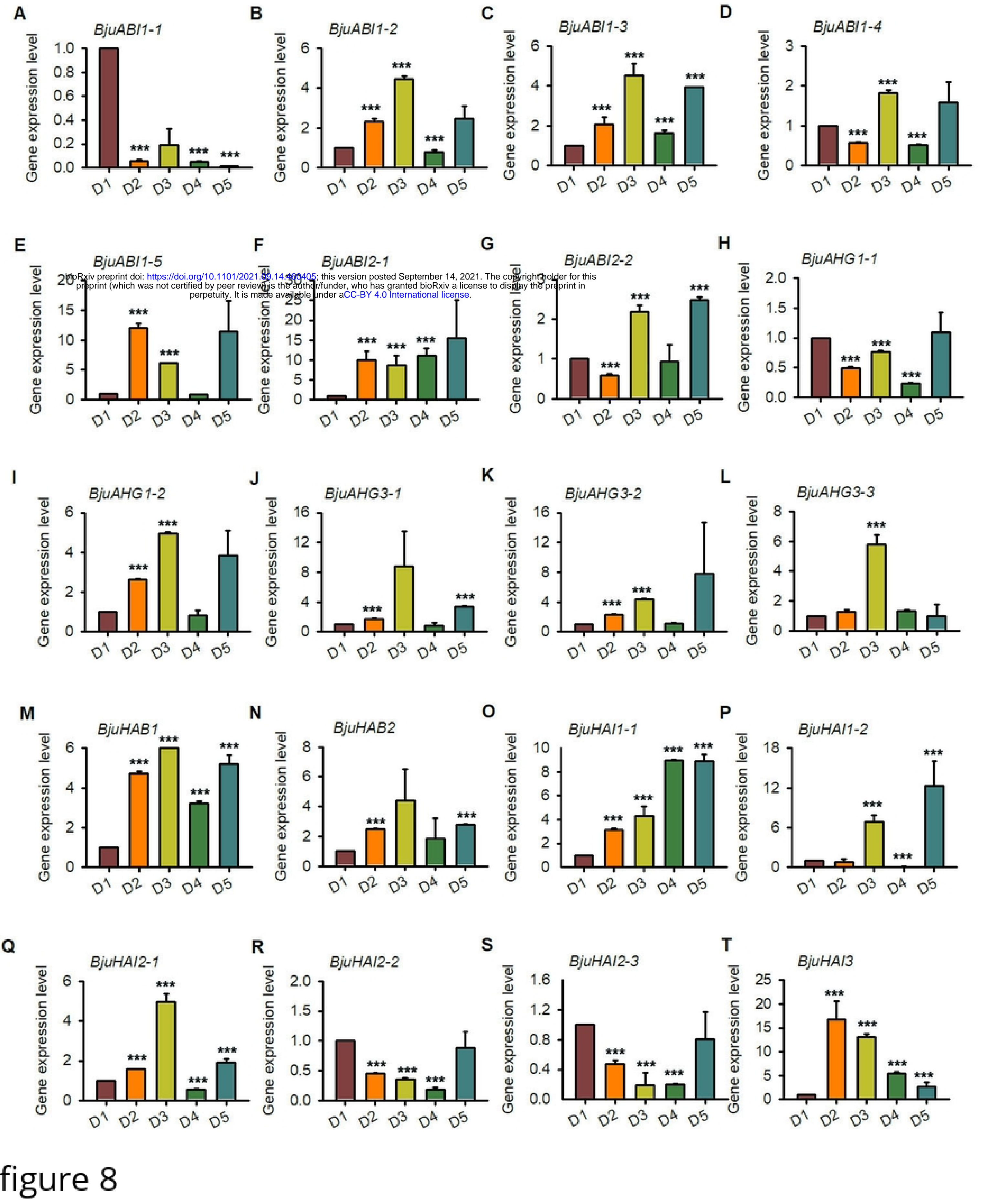


Table 1. The elade A BjuPP2Cs family members in B. juncea var. tumida

\begin{tabular}{|c|c|c|c|c|c|c|c|c|c|c|c|}
\hline Group & Gene name & Lous & Sequence ID & Exon & Start (bp) & End (bp) & Genomics (bp) & CDS (bp) & Protein (aa) & pI & MW (kD) \\
\hline \multirow[t]{5}{*}{$A L A B I I$} & $B j u A B I I-1$ & $\mathrm{~A} 01$ & BjuA004393 & 4 & 9995166 & 9996741 & 1576 & 1248 & 415 & 6.44 & 45.7 \\
\hline & $B j u A B I I-2$ & $\mathrm{~A} 03$ & BjuA013512 & 4 & 33638598 & 33640105 & 1508 & 1233 & 411 & 5.51 & 45.158 \\
\hline & $B j u A B I I-3$ & B05 & BjuB040327 & 4 & 7461353 & 7462895 & 1543 & 1257 & 419 & 5.87 & 45,785 \\
\hline & BjuABII-4 & A08 & BjuA02959I & 3 & 18441423 & 18442925 & 1503 & 975 & 325 & 6.15 & 35.907 \\
\hline & $B j H A B I I-5$ & B03 & BjuB043703 & 3 & 5139792 & 5141317 & 1526 & 1209 & 402 & 5.92 & 44.679 \\
\hline \multirow[t]{2}{*}{$A L A B I 2$} & BjuABI2-I & B02 & BjuB001428 & 8 & 22604047 & 22608170 & 4124 & 1614 & 538 & 5.14 & 63.057 \\
\hline & $B j u A B I 2-2$ & $\mathrm{~A} 10$ & BjuA044909 & 8 & 10610992 & 10616335 & 5344 & 1611 & 537 & 7.48 & 59,467 \\
\hline \multirow[t]{2}{*}{$A L A G I$} & BjuAHGI-I & $\mathrm{A} 03$ & BjuA010184 & 4 & 8253750 & 8255933 & 2184 & 1257 & 418 & 6.08 & 45.533 \\
\hline & BjuAHGI-2 & B08 & BjuB041185 & 4 & 15899473 & 15901555 & 2083 & 1275 & 424 & 6.08 & 46.768 \\
\hline \multirow[t]{3}{*}{$A L A H G 3$} & BjuAHG3-I & A05 & BjuA020514 & 2 & 28490862 & 28492149 & 1288 & 1200 & 400 & 6.04 & 43.061 \\
\hline & BjuAHG3-2 & B07 & BjuB006899 & 3 & 3123674 & 3125019 & 1346 & 1170 & 389 & 6.22 & 42.243 \\
\hline & BjuAHG3-3 & B01 & BjuB023632 & 3 & 42663804 & 42668223 & 4420 & 1818 & 606 & 7.51 & 67.615 \\
\hline$A t H A B I$ & BjuHABI & B03 & BjuB030059 & 3 & 39259177 & 39260975 & 1799 & 1137 & 379 & 4.63 & 40.875 \\
\hline$A t H A B 2$ & $B j u H A B 2$ & B03 & BjuB004338 & 2 & 2791346 & 2793227 & 1882 & 1158 & 386 & 4.40 & 42.644 \\
\hline \multirow[t]{2}{*}{ AtHAII } & BjuHAII-I & $\mathrm{A} 10$ & BjuA038574 & 4 & 11316559 & 11318415 & 1857 & 1236 & 412 & 6.07 & 45.517 \\
\hline & BjuHAII-2 & B02 & BjuB008108 & 4 & 29546261 & 29548082 & 1822 & 1194 & 397 & 7.06 & 44.176 \\
\hline \multirow[t]{3}{*}{ AtHAI2 } & BjuHAI2-I & A09 & BjuA01947I & 3 & 54391632 & 54393366 & 1735 & 1308 & 436 & 6.17 & 48.931 \\
\hline & BjuHAI2-2 & B06 & BjuB015401 & 3 & 17704399 & 17706077 & 1679 & 1263 & 421 & 6.17 & 47.211 \\
\hline & BjuHAI2-3 & $\mathrm{A} 10$ & BjuA037326 & 3 & 782631 & 784079 & 1449 & 1281 & 426 & 5.93 & 47,804 \\
\hline$A t H A I 3$ & BjuHAI3 & Contig & BjuO013253 & 3 & 1153566 & 1155192 & 1627 & 1104 & 368 & 5.50 & 41.009 \\
\hline
\end{tabular}

pI: Isoeleetric point; MW: molecular weight; CDS: coding sequence. 